\title{
The contribution of nearby supernova remnants on the cosmic ray flux at Earth
}

\author{
A. AL-Zetoun ${ }^{1 \star}$, A. Achterberg \\ ${ }^{1}$ Department of Astrophysics, IMAPP, Radboud University, Nijmegen, P.O. Box 9010, 6500 GL Nijmegen, The Netherlands
}

Accepted..., Received...; in original form ...

\begin{abstract}
We consider anisotropic diffusion of Galactic cosmic rays in the Galactic magnetic field, using the Jansson-Farrar model for the field. In this paper we investigate the influence of source position on the cosmic ray flux at Earth in two ways: [1] by considering the contribution from cosmic ray sources located in different intervals in Galacto-centric radius, and [2] by considering the contribution from a number of specific and individual close-by supernova remnants. Our calculation is performed by using a fully three-dimensional stochastic method. This method is based on the numerical solution of a set of stochastic differential equations, equivalent to Itô formulation, that describes the propagation of the Galactic cosmic rays.
\end{abstract}

Key words: Methods: numerical - diffusion - magnetic fields - cosmic rays-supernova remnants

\section{INTRODUCTION}

Galactic cosmic ray (CR) sources, presumably core-collapse type II supernovae, are thought to be concentrated in the Galactic disk. Therefore, the effective source density should have a radial distribution similar to that of type II (core-collapse) supernova remnants (SNRs), see for instance Strong et al. (2007). It is generally believed that these SNRs are thought to be the main source of Galactic CRs (GCRs), see for instance Blandford \& Eichler (1987). Additionally, the strong winds from O-B stars (e.g. Webb et al. (1985) and Thoudam et al. (2016)), neutron stars, see for instance Venkatesan et al. (1997) and pulsar winds may very well contribute to the observed CR flux at Earth. In this study we will disregard these possible CR sources, concentrating on CRs from core-collapse supernovae. As a consequence, in our model the distribution from Type II supernovae determines the CR source distribution. The distribution of Galactic SNRs derived in the literature is poorly constrained for large Galacto-centric radii. Observations have found that the distribution of SNRs extends approximately up to a radial distance of $R \leq 16 \mathrm{kpc}$, assuming that the SNR distribution is grosso mode axi-symmetric with respect to the Galactic Center Case \& Bhattacharya (1996).

In a simple Leaky Box Model, where the typical CR residence time in the Galaxy is determined by the height $H_{\mathrm{cr}} \simeq 4-10 \mathrm{kpc}$ of the CR halo above the Galactic disk and by the typical CR diffusion coefficient $D_{\mathrm{cr}}$ as $t_{\mathrm{res}} \simeq H_{\mathrm{cr}}^{2} / 2 D_{\mathrm{cr}}$, the contribution to the observed CR flux from sources at a distance from the observer much larger than $H_{\mathrm{cr}}$ rapidly becomes negligible. For instance, in the case of isotropic diffusion with instantaneous escape at $z= \pm H_{\mathrm{cr}}$, the CR density in the Galactic mid-plane due to a source at a give distance $\varpi$ decays for $\varpi \gg H_{\text {cr }}$ as (see Appendix):

$$
n_{\mathrm{cr}}\left(\varpi, 0, t=\frac{\varpi H_{\mathrm{cr}}}{\pi D_{\mathrm{cr}}} \tau\right) \propto \frac{\pi D_{\mathrm{cr}}}{\varpi H_{\mathrm{cr}} \tau} \exp \left(-\frac{\pi \varpi}{4 H_{\mathrm{cr}}} \tau\right)
$$

It is assumed that CRs are injected impulsively at $t=0$, see the Appendix for details. Here the dimensionless time $\tau$ is given

* E-mail: a.al-zetoun@astro.ru.nl 
by $\tau \equiv \pi D_{\mathrm{cr}} t / \varpi H_{\mathrm{cr}}$, with $\tau \simeq 1$ corresponding to moment of the the maximum CR density at given $\varpi \gg H_{\mathrm{cr}}$ as a function of time.

The effect of nearby discrete sources to the observed CR nuclei has been studied extensively in a number of different models. For instance Erlykin \& Wolfendale (2006), studied the anisotropy observed at Earth from local sources located within a distance of few kpc from the Sun. They considered sources that are distributed randomly in the nearby Galaxy. They concluded that the anisotropy of arrival directions is produced mainly by these local sources. Strong \& Moskalenko (2001) used the GALPROP code to study the effect of the discrete sources of SNRs on the CR proton densities in the Galaxy. Thoudam (2007) studied the CR density fluctuations from a known nearby source located within a distance of $1.5 \mathrm{kpc}$ from Earth, and the influence of the nearby SNRs on the observed CR anisotropy below the knee in the CR spectrum.

The propagation of the GCRs from their source to the observer is usually described in the diffusion approximation. In general, the diffusion of charged CRs is thought to be due to the deflection by stochastic Lorentz forces, either due to irregularities in the Galactic magnetic field, which is known to have a strong stochastic component, or due to self-excited Alfvén waves, see for instance Berezinskii et al. (1990) and Strong et al. (2007).

Several quasi-analytical models have been proposed to describe CR propagation in the Galaxy as a whole. The simplest such model is the Leaky Box Model that considers the global effects of CR propagation inside the Galaxy, and escape from the Galaxy. It was proposed by Cowsik \& Wilson (1973) as an improvement on the simpler slab model Lezniak (1976) that was in use at that time. The Leaky Box Model considers the Galaxy as a box with transparent walls around the Galactic disk. The CR sources are distributed uniformly in the Galactic disk, and the CRs diffuse within the Box, escaping as they reach it is upper and lower edge. Generally speaking, the Leaky Box Model satisfactorily reproduces existing data on stable primary and secondary nuclei in CRs, but with some limitations, see for instance Strong et al. (2007).

Nowadays, various widely-used numerical codes are available that concurrently calculate the propagation and CR interactions (such as losses, spallation in the interstellar gas, re-acceleration etc.) of the Galactic CRs in the Galaxy. Examples of such codes are GALPROP, see Strong et al. (2010), DRAGON, see Evoli et al. (2008) and CRPropa 3.1, see Merten et al. (2017), as well as PICARD Kissmann (2014). GALPROP code is the most widely code and has been developed to overcome the limitations of analytical and semi-analytical models like the Leaky Box model. The code calculates the transport and interactions of the propagation of CR nuclei, electrons and positrons, anti-protons. However, it has not yet implemented a method for dealing with anisotropic diffusion. DRAGON code is a numerical tool designed to cover all relevant processes include GCRs and their secondary products over a very wide energy range, that addresses for example the anisotropic diffusion problem. The recent CRPropa update CRPropa 3.1 also solves the CR spatial transport equation using stochastic differential equations, the method also used in this paper. This is the most straightforward way to treat anisotropic CR diffusion with respect to an arbitrary magnetic field. Finally, the PICARD code is a more sophisticated numerical tool that uses advanced contemporary numerical algorithms for solving the diffusion equation that compute a steady state solution for the CRs propagation problem without any modification of numerical parameters (such as energy dependence of diffusion coefficients or parameters associated with the nuclear network that is used to calculate CR spallation) by the user.

The outline of this paper is as follows: In Section 2, we present the propagation model and the relevant assumptions, like the source distribution of CR, the diffusion tensor and it is connection to the large scale Galactic magnetic field. In Section 3 we present the spatial distribution of CRs from a uniform source as well as from known SNRs, and discuss the results. We do this for two values for the ratio of the parallel - and perpendicular diffusion coefficients: $D_{\perp} / D_{\|}=1$ (isotropic diffusion) and $D_{\perp} / D_{\|}=0.01$ (strongly anisotropic diffusion). In Section 4 we present the conclusions.

\section{MODEL DESCRIPTION}

Recently, AL-Zetoun \& Achterberg (2018) have proposed a fully three-dimensional model for CR propagation in the Galactic magnetic field, based on the numerical solution of a set of stochastic differential equations in the Itô formulation. We solve these equations using a fully-anisotropic diffusion tensor, the situation that is expected in the presence of a large-scale ordered Galactic magnetic field.

The CRs are represented by test particles following a trajectory $\boldsymbol{x}(t)$ that change their position by an amount $\Delta \boldsymbol{x}$ in a time-span $\Delta t$ according to the prescription: 


$$
\Delta \boldsymbol{x}=\boldsymbol{V} \Delta t+\Delta \boldsymbol{x}_{\mathrm{diff}} .
$$

The vector $\boldsymbol{V}$ is a mean velocity, formally defined as:

$$
\boldsymbol{V}=\boldsymbol{U}+\boldsymbol{\nabla} \cdot \mathbf{D}
$$

in component notation $V_{i}=U_{i}+\partial D_{j i} / \partial x_{j}$, with $\boldsymbol{U}$ the bulk velocity of the (ionized) interstellar medium and $\mathbf{D}$ the CR diffusion tensor. For anisotropic diffusion with respect to the large-scale field (see Section 2.2 for more details), with a diffusion tensor $\mathbf{D}=D_{\|} \hat{\boldsymbol{b}} \hat{\boldsymbol{b}}+D_{\perp}(\mathbf{I}-\hat{\boldsymbol{b}} \hat{\boldsymbol{b}})$ where $\hat{\boldsymbol{b}}$ is the unit vector along the magnetic field and $\mathbf{I}=\operatorname{diag}(1,1,1)$ is the unit tensor, the diffusive step $\Delta \boldsymbol{x}_{\text {diff }}$ equals in the Itô formulation:

$$
\Delta \boldsymbol{x}_{\mathrm{diff}}=\sqrt{2 D_{\perp} \Delta t} \xi_{1} \hat{\boldsymbol{e}}_{1}+\sqrt{2 D_{\perp} \Delta t} \xi_{2} \hat{\boldsymbol{e}}_{2}+\sqrt{2 D_{\|} \Delta t} \xi_{3} \hat{\boldsymbol{e}}_{3}
$$

Here we employ local Cartesian coordinate system with base vectors $\hat{\boldsymbol{e}}_{1}, \hat{\boldsymbol{e}}_{2}, \hat{\boldsymbol{e}}_{3}$ with the local large-scale magnetic field along $\hat{\boldsymbol{e}}_{3}=\hat{\boldsymbol{b}}$. In recipe (4) the quantities $\xi_{1}, \xi_{2}$ and $\xi_{3}$ are three independent (that is: statistically uncorrelated) stochastic variables, with zero mean and unit dispersion, i.e. in simplified notation: $\langle\xi\rangle=0$ and $\left\langle\xi^{2}\right\rangle=1$. For more details about the model see AL-Zetoun \& Achterberg (2018). Note that $\sqrt{2 D_{\perp} \Delta t}$ and $\sqrt{2 D_{\|} \Delta t}$ are the rms diffusion distances for a timespan $\Delta t$ in a given direction, either perpendicular to the large-scale magnetic field or along the magnetic field.

In what follows we put $\boldsymbol{V}=0$, thereby only including diffusive process and neglecting the effects of a possible Galactic wind, curvature and gradient drift of CR guiding centers in the Galactic magnetic field for simplicity. These effects will be considered in a follow-up paper. We also limit ourselves to a parameter study, focusing on the effect of using different values for the ratio of the perpendicular and parallel diffusion coefficients, $D_{\perp} / D_{\|}$. We take $D_{\perp} / D_{\|} \equiv \epsilon=0.01,1.0$. In the last case we formally recover isotropic diffusion in three dimensions. In the simulation, we remove particles once they reach the upper/lower boundary of the CR halo at $z= \pm H_{\mathrm{cr}}$, corresponding to free escape. This escape determines their residence time $t_{\text {res }}$ in the Galaxy.

\subsection{The distribution of cosmic ray sources}

In our previous work ( AL-Zetoun \& Achterberg (2018)) we described the diffusion of CRs originating from a single point source that was placed at various locations in the Galactic mid-plane $z=0$. We injected the particles impulsively (that is: in a burst of negligible duration) at a fixed Galacto-centric radius close to that of the Sun, which is at $R_{\odot}=8.5 \mathrm{kpc}$. We assumed a uniform diffusion coefficients $D_{\perp}$ and $D_{\|}$, using typical values for $D_{\|}$derived from the (isotropic) Leaky Box Model and the aforementioned simulations for $\mathrm{CR}$ energies of $\sim 1 \mathrm{GeV} /$ nucleon.

In the present paper we investigate the flux near Earth from multiple CR sources. We do this using two different approaches:

(i) First we consider the flux due to multiple sources in a fixed interval in Galacto-centric radius. In this case CRs originate in a ring in the mid-plane of the Galactic disk, centered on the Galactic Center. We do not use discrete source positions, but assume a smooth distribution for the source locations in azimuth with respect to the Galactic Center. We inject the CR particles in the mid-plane $(z=0)$ of the Galactic disk and distribute the source locations uniformly and randomly in four rings around the Galactic Center, with cylindrical injection radius $r_{\text {inj }}$ in the four intervals (in kpc) $2<r_{\text {inj }} \leq 4,4<r_{\text {inj }} \leq 6$, $6<r_{\text {inj }} \leq 8$ and $8<r_{\text {inj }} \leq 10$. The distribution in cylindrical radius $r_{\text {inj }}$ is obtained for each injected particle from the simple prescription:

$$
r_{\mathrm{inj}}=\sqrt{\left(r_{\max }^{2}-r_{\min }^{2}\right) \Xi+r_{\min }^{2}} .
$$

The two radii $r_{\min }$ and $r_{\max }$ are the inner and outer radius of each ring and $\Xi$ is a random variable distributed uniformly between 0 and 1 . The azimuthal angle $\phi$ of the injection site (where $\phi$ is the cylindrical polar coordinate in the plane of the Galactic Disk with respect of the Galactic Center) is chosen randomly from a uniform (top-hat) distribution between 0 and $2 \pi$. This prescription guarantees a uniform (projected) surface density of sources in the Galactic plane. In this case all CRs are injected at time $t=0$ and followed until they escape the Galaxy as they reach a height $H_{\mathrm{cr}}$ above/below the Galactic mid-plane. 
(ii) We look at the contribution of a number of specific sources close to Earth. We first consider four hypothetical equidistant sources (distance: $5 \mathrm{kpc}$, see Figure 5 (c)) in order to study how the local magnetic field geometry influences their propagation to Earth. Next we consider CRs from the same set of sources as employed by Thoudam (2007), see Table 1 in Section 3.3. These CRs are released from their sources at the estimated time of the associated supernova explosion, see below for a discussion of this assumption. In the second case we consider only known SNRs located within a distance of $1.5 \mathrm{kpc}$ from the Solar System, with an age $\leq 2.4 \times 10^{4} \mathrm{yr}$. This age is much smaller than the typical residence time of CR in the Galaxy, which is $t_{\mathrm{res}} \simeq 10^{7} \mathrm{yr}$ in these simulations. This means that most of the CRs produced in these sources are still diffusing in the Galaxy. We note in passing that the residence time is set by the assumed value of $D_{\|}$with $t_{\text {res }} \propto D_{\|}^{-1}$, and can be re-scaled to different values by changing that parameter.

These local sources are expected to produce temporal significant variations in the CR densities at Earth, see the discussion in Thoudam (2006). Table 1 shows the distance of each known SNRs from the Solar System, their exact position in Cartesian coordinates $(x, y)$ in the mid-plane centered on the Galactic Center, with the Solar System at $x=-R_{\odot}=-8.5 \mathrm{kpc}$, Galactic longitude $l$, as well as the age as given in Thoudam (2007). More details regarding these sources can be found in the SNR catalog of Green (2017).

In both cases we define a local volume around the Sun with a radius of $1 \mathrm{kpc}$. We record each time step how many CRs reside inside this volume and add the result over the different steps during the entire run of the simulation. We return to the reason for this approach below. We use as a criterion for 'residence' $\left|\boldsymbol{r}_{\mathrm{CR}}-\boldsymbol{r}_{\odot}\right|<1 \mathrm{kpc}$, with $\boldsymbol{r}_{\mathrm{cr}}$ the position of a CR and $\boldsymbol{r}_{\odot}$ that of the Sun.

The CRs in the vicinity of the Solar System diffuse in a halo around the Galactic disk with height $H_{\text {cr. }}$ The estimated value for $H_{\mathrm{cr}}$ lies in the range $(4-10) \mathrm{kpc}$, see for instance Strong et al. (2010). CRs escape at the upper (lower) halo boundary, i.e. at $z=+H_{\mathrm{cr}}\left(z=-H_{\mathrm{cr}}\right)$. The local volume around the Solar System, defined here, is small enough that edge effects due to $\mathrm{CR}$ escape play no role, except in determining the local CR density. We take the CR density inside this local volume, centered on the position of the Solar System, as a measure for the observed CR flux at Earth.

We neglect the effects of the Solar Wind on the observed CR flux. We also neglect the effects of nuclear spallation of CRs in the interstellar gas.

\section{$2.2 \quad$ Anisotropic Diffusion}

The presence of a large-scale (regular) magnetic field clearly breaks isotropy as the field introduces a preferred direction. This is important for the calculations presented here as this will influence the contribution from different sources to the local CR density. Quite generally, one expects diffusion along the field to be more rapid than diffusion across the field. As demonstrated in AL-Zetoun \& Achterberg (2018), the ratio of the perpendicular and parallel diffusion coefficients, $\epsilon \equiv D_{\perp} / D_{\|}$, see also Eqn. 6 below, regulates the average residence time for the CR population in the Galaxy in the Jansson-Farrar field (see Section 2.3 ) we adopt. It increases strongly for small values of $\epsilon$. When considering the local CR flux entering the Solar System one expects that sources that are 'magnetically connected', that is: sources on or close to those field lines of the regular field that cross the local volume, will tend to have a larger contribution to the local CR density than 'unconnected' sources.

For anisotropic diffusion in a large-scale Galactic magnetic field true diffusion (as opposed to drift) is characterized by a diffusion tensor $\mathbf{D}$ that needs to take into account of the different values for the perpendicular and parallel diffusion coefficients. The diffusion tensor is given by:

$$
\mathbf{D}=D_{\perp}(\mathbf{I}-\hat{\boldsymbol{b}} \hat{\boldsymbol{b}})+D_{\|} \hat{\boldsymbol{b}} \hat{\boldsymbol{b}}=D_{\|}[\epsilon(\mathbf{I}-\hat{\boldsymbol{b}} \hat{\boldsymbol{b}})+\hat{\boldsymbol{b}} \hat{\boldsymbol{b}}]
$$

with $\mathbf{I}$ the $3 \times 3$ unit tensor, $\boldsymbol{B}$ is the ordered magnetic field, $\hat{\boldsymbol{b}} \equiv \boldsymbol{B} /|\boldsymbol{B}|$ is the unit vector along the magnetic field and $\hat{\boldsymbol{b}} \hat{\boldsymbol{b}}$ is the associated dyadic tensor with components $(\hat{\boldsymbol{b}} \hat{\boldsymbol{b}})_{i j}=\hat{b}_{i} \hat{b}_{j}$. In fact, we assume that the diffusion along the large-scale field (governed by the parallel diffusion coefficient $D_{\|}$) and the diffusion across the field (governed by the perpendicular diffusion coefficient $D_{\perp}$ ) may reasonably depend only on the micro-physics of the ( axi-symmetric) diffusion on in a locally field-aligned coordinate system. In this paper we consider spatially uniform diffusion coefficients $D_{\perp}$ and $D_{\|}$, where $D_{\perp}$ and $D_{\|}$have an energy dependence of the form that usually described by empirical diffusion models, see for instance Berezinskii et al. (1990) and Strong et al. (2007). Typically, for CRs with energy $E$ and charge number $Z$ isotropic diffusion models give a diffusion coefficient of order 


$$
D(E)=D_{0}\left(\frac{E / Z}{1 \mathrm{GeV} / \text { charge }}\right)^{\delta},
$$

where $D_{0}=3 \times 10^{28} \mathrm{~cm}^{2} \mathrm{~s}^{-1}$ and $\delta \simeq 1 / 3$.

In our calculation we adopt this scaling for both $D_{\|}$and $D_{\perp}=\epsilon D_{\|}$since we assume that $\epsilon$ is constant. As long as one considers particles of a single energy/nucleon this is not really a problem as a different scaling with energy of $D_{\perp}$ and $D_{\|}$can be absorbed into the value of $\epsilon$.

There is one case in which the assumption of a constant $\epsilon$ can be justified for different CR energies as $D_{\perp} \propto D_{\|}$. This is the case where the random magnetic field component $\delta \boldsymbol{B}_{\perp}$ perpendicular to the regular magnetic field has a correlation length smaller than the scattering mean free path $\lambda_{\|}$along the field. Note that by definition $D_{\|}=\lambda_{\|} c / 3$ for relativistic CRs. If one has $D_{\perp}^{\mathrm{t}} \ll D_{\|}$with $D_{\perp}^{\mathrm{t}}$ the 'ordinary' cross-field diffusion coefficient due to very small-scale turbulence, the tilt of the total field $\boldsymbol{B}=\boldsymbol{B}_{0}+\delta \boldsymbol{B}_{\perp}$ with respect to the regular field $\boldsymbol{B}_{0}$ leads to an effective cross-field diffusion coefficient (again with respect to the regular field) of order:

$$
D_{\perp}^{\mathrm{eff}} \simeq D_{\|}\left(\frac{\left|\delta \boldsymbol{B}_{\perp}\right|}{B_{0}}\right)^{2}
$$

This result holds provided the correlation length of the random field component along the regular field is sufficiently small, see the careful discussion in Isichenko (1991) for the conditions needed for the validity of this result.

In the calculations presented here we do not assign an energy to the particles, but take typical values for CRs with $E / A \sim$ $1 \mathrm{GeV}$ per nucleon.

Experimental studies of the B/C ratio and the sub-Fe/Fe ratio suggest that $\delta$ changes between ( 0.6 to 0.3$)$ in the $\mathrm{GeV}-\mathrm{TeV}$ region. The value of $D_{0}$ is only weakly constrained by measurements of the observed flux of stable nuclei. For example: unstable/stable ratios $\left(B^{10} / B^{9}\right)$ need large CR halo $H_{\mathrm{cr}} \simeq 5 \mathrm{kpc}$, which leads to large values of $D_{0} \simeq(3-5) \times 10^{28} \mathrm{~cm}^{2} \mathrm{~s}^{-1}$ at $E_{0}=1 \mathrm{GeV}$ per nucleon, see for instance Strong et al. (2007) and Evoli et al. (2008).

\subsection{Model for the large-scale Galactic magnetic field}

We employ the GMF model of Jansson \& Farrar (2012a), and Jansson \& Farrar (2012b) (henceforth: the JF model) to describe the large-scale GMF. These authors use a Galacto-centric $(r, \phi, z)$, as well as a right-handed Cartesian $(x, y, z)$ coordinate system, with the Sun located along the negative $x$-axis, at $x_{\odot}=-8.5 \mathrm{kpc}$. The Galactic north is in the positive $z$-axis, and $\phi=0$ is along the positive $x$-axis. The magnetic field is set to zero for $r>20 \mathrm{kpc}$.

The JF model is a three-component model consisting of a disk component, a toroidal component, and the so-called $X$-field:

(i) The disk component is described by a toroidal field in the $x-y$ plane that includes a molecular ring between $3-5 \mathrm{kpc}$, which is purely azimuthal with a constant field strength, and a logarithmic spiral arm structures (eight arms in total) at larger radii up to $20 \mathrm{kpc}$, where the magnetic field has a different value in each arm.

(ii) The halo component has a purely toroidal (azimuthal) component with different radial and vertical extent in the northern and southern halo.

(iii) The $X$-field component is purely poloidal and axi-symmetric, (i.e. no azimuthal component). Given field lines are straight, with an inclination angle determined solely by the cylindrical radius $r_{\mathrm{p}}$ where the field crosses the Galactic midplane.

For all detailed description of the model and it is parameters we refer to, see Jansson \& Farrar (2012a), and Jansson \& Farrar (2012b) and references therein, as well as the discussion in Section 2 of AL-Zetoun \& Achterberg (2018). 


\section{$6 \quad$ AL-Zetoun, Achterberg}

\subsection{Grammage and the density of the diffuse interstellar medium}

CRs accumulate grammage $\Sigma_{\text {cr }} \sim$ (pathlength) $\times$ (mass density) as they traverse the Galactic disk. This grammage is an important quantity as it determines the number of spallation reactions that a typical primary CR undergoes. In our calculation the grammage is accumulated each time step and given by:

$$
\Delta \Sigma_{\mathrm{cr}}=\rho\left(\boldsymbol{r}_{\mathrm{cr}}\right) v \Delta t
$$

where $\rho$ is the density of the diffuse gas, $v=c \sqrt{1-\left(m c^{2} / E\right)^{2}}$ is the velocity of a CR with rest mass $m$ and energy $E$ and $\boldsymbol{r}_{\mathrm{cr}}$ is the instantaneous position of the CR inside the Galaxy. In our calculation, we keep $E$ constant, and consider particles with $E \gg m c^{2}$ so that $v \simeq c$.

The density of the diffuse gas in the disk mid-plane of the Galaxy (i.e. at $z=0$ ) from the relative abundance of secondary CRs scales with radius $r$ as, see for instance Kalberla \& Kerp (2009):

$$
\rho(r, 0)= \begin{cases}\rho_{0} & \text { for } r<R_{\mathrm{c}}=7 \mathrm{kpc} \\ \rho_{0} \exp \left[-\left(r-R_{\mathrm{c}}\right) / R_{\mathrm{d}}\right] & \text { for } r>R_{\mathrm{c}}=7 \mathrm{kpc} .\end{cases}
$$

The scale length in the exponential is $R_{\mathrm{d}} \simeq 3.15 \mathrm{kpc}$, and $\rho_{0} \simeq 3 \times 10^{-24} \mathrm{~g} \mathrm{~cm}^{-3}$. The typical thickness $H_{\mathrm{d}}$ of the hydrogen disk flares out as $r$ increases: it scales as: It is expected that this law will break down for $r<5 \mathrm{kpc}$, but we will use it anyway for lack of a better model. In view of this we can adopt an axi-symmetric density distribution that scales with height $|z|$ above the mid-plane as:

$$
\rho(r, z)=\rho(r, 0) \exp \left(-\frac{|z|}{H_{\mathrm{d}}(r)}\right)
$$

The typical thickness $H_{\mathrm{d}}$ of the hydrogen disk flares out as $r$ increases: it scales as:

$$
H_{\mathrm{d}}(r)=H_{0} \exp \left(r / R_{\mathrm{h}}\right)
$$

with $R_{\mathrm{h}} \simeq 9.8 \mathrm{kpc}$ and $H_{0} \simeq 0.063 \mathrm{kpc}$. It is expected that this law will break down for $r<5 \mathrm{kpc}$, but we will use it anyway for lack of a better model.

\section{THE SIMULATIONS}

In this Section we describe the results of the diffusion of CRs origination from multiple sources at different Galacto-centric radii for two approaches. In the first case, we inject the CR particles uniformly and randomly in the mid-plane of the Galactic disk, at fixed Galacto-centric radius close to the Sun in four rings, assuming a smooth distribution of source locations in azimuth with respect to the Galactic center. In the second case, we inject the CR particles from discrete sources listed in table 1 in the mid-plane of the Galactic disk, as well as from discrete sources at a certain distance from the Sun. In all cases, particles are injected at time $t=0$ from their sources. We then let the CRs diffuse according to prescription (4), assuming uniform diffusion coefficients $D_{\perp}$ and $D_{\|}$, as well as no mean flow. The flux and the accumulated grammage are calculated in the local volume around the Solar System from multiple sources at different Galacto-centric radii.

We want to emphasize that the distribution of CR ages obtained by this method for the CRs inside the local volume centered on the Solar System is not the escape time from the Galaxy, but the CR age during residence inside the local volume. This is always significantly less than the escape time. The escape time $t_{\text {res }}$ is the total time that a CR spends in the Galaxy after injection.

\subsection{Results for CRs from multiple sources in four Galacto-centric rings}

To investigate the effects of the strength of perpendicular diffusion on the CR flux at Earth from multiple sources we have simulated the propagation of CRs for sources that reside in four rings, with a cylindrical radius $r_{\text {inj }}$ (in kpc) between 
$2<r_{\text {inj }}<4,4<r_{\text {inj }}<6,6<r_{\text {inj }}<8$ and $8<r_{\text {inj }}<10$. In each case we use two values for the ratio of perpendicular and parallel diffusion coefficients: $\epsilon=D_{\perp} / D_{\|}=0.01$, the case of weak diffusion across the magnetic field where the diffusive step perpendicular to the field $\left(\propto \sqrt{D_{\perp}}\right)$ is ten times smaller than the step along the field, and $\epsilon=1$, the case of isotropic diffusion where the magnetic field has no influence. The parallel diffusion coefficient is kept constant at $D_{\|}=3 \times 10^{28} \mathrm{~cm}^{2} \mathrm{~s}^{-1}$. The thickness of the CR halo above the disk is taken to be $H_{\mathrm{cr}}=4 \mathrm{kpc}$. CRs escape freely once they reach $z= \pm H_{\mathrm{cr}}$.

Figure 1 (left column) shows the spatial distribution of the CRs in the Galactic disk at the moment of escape, projected onto the disk mid-plane. CRs are injected randomly in the four rings with a uniform distribution per unit disk area. The CRs are recorded once they reach upper (lower) boundary of the CR halo above the Galactic disk, located at $z=+H_{\mathrm{cr}}\left(z=-H_{\mathrm{cr}}\right)$, or when they reach the edge of the Galaxy, taken to be at a Galacto-centric distance equal to $r=r_{\max }=20 \mathrm{kpc}$.

The right column of Figure 1 shows, for each of the rings, the disk-projected density of all CRs recorded inside the local volume around the Solar System during the entire simulation. We neglect the height of the Sun $\left(z_{\odot} \simeq 20\right.$ pc $)$ above the plane. The asymmetry in this distribution reflects the position of the Sun with respect to the rings.

Figure 2 is the same as Figure 1, but now for strongly anisotropic diffusion with $\epsilon=D_{\perp} / D_{\|}=0.01$. As one can see in the right column, top to down: the number of CRs inside the local volume decreases when the sources (on average) are located farther away from the Sun. This effect becomes more pronounced for small $r_{\text {inj }}<r_{\odot}=8.5 \mathrm{kpc}$. Here we see the influence of the fact that only those parts of the ring contribute to the CR density that are magnetically connected to the local volume by the spiral disk field in the JF model, since diffusion across the field is much slower than diffusion along the field in this case.

In both cases we show the results for a fixed number of simulated CRs (in this case $10^{5}$ in each simulation) from each ring. If SNRs are distributed uniformly per unit disk area, the absolute number of CRs produced in each ring is proportional to the ring area, $\pi\left(r_{\max }^{2}-r_{\min }^{2}\right)$, if $r_{\min }<r_{\text {inj }}<r_{\max }$. This determines the relative contributions from the different rings to the total number of CRs at a given location. That will be considered below in Figure 4. Below we will also relax the assumption that the SNRs producing the CRs are distributed uniformly over the Galactic disk.

In Figure 3 we compare the distribution of total residence time in the Galaxy (the dashed lines) to the distribution of CR ages inside the local volume (the solid lines). We do this separately for CRs coming from each of the four rings. The black distribution shows the total number of CRs from all four rings together.

What is shown here (and in the following figures) is the distribution over CR age that is obtained when one sums over the entire duration of the simulation. This essentially corresponds to an integration over time of the CR number inside the local volume. This is equivalent to the mean distribution that one would observe (at a given time) for a continuous and constant CR production rate in the Galaxy. The simulation runs long enough for all CRs created at the start of the simulation to have escaped the Galaxy. As before we take $H_{\mathrm{cr}}=4 \mathrm{kpc}$ for the thickness of the CR halo and $\epsilon=D_{\perp} / D_{\|}=$ 1(upper plot), 0.01(lower plot).

The distributions shown in Figure 3 are normalized distributions, that is: they do not take account of the different absolute number of CRs produced in each individual ring. This is perhaps most obvious in the residence time distributions (the dashed histograms). These are virtually identical for each of the four rings. That result is to be expected since [1] the height $H_{\mathrm{cr}}$ of the escape boundary above the Galactic mid plane is taken to be independent of Galacto-centric radius $r$, [2] the diffusion coefficients are assumed to be constant and [3] the disk component of the spiral disk field in the JF magnetic field model has a constant pitch, independent of radius.

In the case of isotropic diffusion, the residence time distribution and the distribution of CR age are very similar in shape, in agreement with the notion that CRs cross the Galactic disk many many times before escaping. Of course the age of CRs inside the local volume is always less than the total residence time. The contribution of the ring $8<r_{\text {inj }}<10 \mathrm{kpc}$, which contains the Solar System, and the adjacent ring $6<r_{\text {inj }}<8 \mathrm{kpc}$ show a significant increase of CRs with an age below 10 Myr in the local volume: these are CRs injected close to the Sun that are able to reach the local volume in a relatively short time. For the rings with $r_{\mathrm{inj}}<6 \mathrm{kpc}$ this effect is not present.

In the case of strongly anisotropic diffusion with $\epsilon=0.01$, the age distribution (the solid lines) is very flat until about $10^{7} \mathrm{yr}$, for larger ages the number of particles decreases rapidly. In contrast: for $\epsilon=1$ the number of CRs decreases continuously with increasing age. The typical CR age is now also longer than in the case $\epsilon=1$, as for most of the disk it is regulated mostly by $D_{\perp}$ rather than $D_{\|}$so that $t_{\text {res }} \simeq H_{\mathrm{cr}}^{2} / 2 D_{\perp}$, see also AL-Zetoun \& Achterberg (2018).

This result can be quantified for CRs originating close to the Sun, at $r=R_{\odot}=8.5 \mathrm{kpc}$. Escape is mainly through diffusion in the vertical ( $z$-direction), with an effective diffusion coefficient equal to:

$$
D_{z z}=\hat{\boldsymbol{z}} \cdot \mathbf{D} \cdot \hat{\boldsymbol{z}}=D_{\|}\left[b_{z}^{2}+\epsilon\left(1-b_{z}^{2}\right)\right]
$$


Here $b_{z}=B_{z} / B$ is the component of the unit vector along the magnetic field in the $z$-direction. Using the numbers in Jansson \& Farrar (2012a): near the Sun one has a spiral disk field with strength equal to $B_{\text {disk }}=1.18 \mu \mathrm{G}$ and pitch angle $p=11.5$ degrees, an $X$-field in the $r-z$ plane with a strength of $B^{\mathrm{X}} \simeq 0.25 \mu \mathrm{G}$ and a field elevation angle of $i_{0}=49$ degrees. This implies $B_{r}=B_{\text {disk }} \sin p+B^{X} \cos i_{0} \simeq 0.40 \mu \mathrm{G}, B_{\phi}=B_{\text {disk }} \cos p \simeq 1.16 \mu \mathrm{G}$ and $B_{z}=B^{\mathrm{X}} \sin i_{0} \simeq 0.19 \mu \mathrm{G}$. Then $B \simeq 1.24 \mu \mathrm{G}$ and $b_{z}=B_{z} / B \simeq 0.11$ so that:

$$
D_{\mathrm{zz}}=D_{\|}[0.023+0.976 \epsilon] \simeq 0.033 D_{\|}
$$

where the last equality is for $\epsilon=0.01$. In that case the effective residence time increases with respect to the case of isotropic diffusion to $t_{\mathrm{res}}=H_{\mathrm{cr}}^{2} / 2 D_{z z} \simeq 30 H_{\mathrm{cr}}^{2} / 2 D_{\|}$, in good accordance with the results of the simulations shown in Figure 3 .

The distribution of $\mathrm{CR}$ age of $\mathrm{CRs}$ inside the local volume no longer resembles the residence time distribution. For the two inner rings, $2<r_{\mathrm{inj}}<4 \mathrm{kpc}$ and $4<r_{\mathrm{inj}}<6 \mathrm{kpc}$ (the green and yellow histograms respectively), CRs take much longer to reach the local volume as they have to diffuse a considerable distance across the small-pitch JF disk magnetic field spiral. This explains the absence of particles from these rings at the lower end of the age distribution. CRs originating from the ring with $6 \mathrm{kpc}<r<8 \mathrm{kpc}$ (red histogram) have an age distribution that is very flat until $(2-3)$ Myr, decaying rapidly thereafter. CRs injected from $8 \mathrm{kpc}<r<10 \mathrm{kpc}$ show a strong decrease with increasing age, which is the combined effect of the pitch of the disk field and of the shape of the X-field which transports particles to larger Galacto-centric radii through parallel diffusion along the resultant magnetic field. In all cases the CRs in the local volume are now considerably younger than escaping particles, much more so than in the case of isotropic diffusion.

Summarizing: both the residence time distribution and the age distribution of CRs in the local volume depend rather strongly on the diffusion coefficient ratio $\epsilon=D_{\perp} / D_{\|}$. When $D_{\perp} / D_{\|}=1$ the particles diffuse relatively fast, and need less time to reach Earth or escape the Galaxy. In this case, the two distributions have a similar shape. When $D_{\perp} / D_{\|}=0.01$ CRs reside $\sim 50 \times$ longer in the Galaxy. The escape time distribution and the age distribution in the local volume no longer resemble each other, and the typical age of CRs in the local volume is considerably less than the residence time. CRs originating from $r_{\text {inj }} \leq 6 \mathrm{kpc}$ are only able to reach the local volume after (typically) $10 \mathrm{Myr}$.

Figure 4 shows the absolute number of simulated CRs from each of the four rings found over the entire simulation inside the local volume as a function of CR age. Here we assume a source density per unit disk area so that the number of CRs produced in each ring is proportional to the ring area $\pi\left(r_{\max }^{2}-r_{\min }^{2}\right)$. The black distribution is the total number of CRs from all four rings, which is what determines the local CR flux. The parameters of the simulation are the same as before.

In the case of isotropic diffusion $(\epsilon=1)$ one clearly sees that the inner rings contribute less to the local CR density at given $\mathrm{CR}$ age. In the anisotropic case $(\epsilon=0.01)$ the contributions from each of the rings are very distinct due to the effects of the magnetic field geometry on CR propagation.

Figures 5 and 6 are similar to Figure 4. Here we assume that the surface density $N(r)$ of CR sources (SNRs) scales with Galacto-centric radius $r$ as:

$$
N_{\mathrm{snr}}(r) \propto \exp \left(-\frac{r}{R_{\mathrm{snr}}}\right)
$$

The exponential scale length $R_{\mathrm{snr}}$ is chosen to be $R_{\mathrm{snr}}=5.4 \mathrm{kpc}$ in Figure 5 and equal to $R_{\mathrm{snr}}=8 \mathrm{kpc}$ in Figure 6 . All simulated CRs are given a weight $\propto N\left(r_{\text {inj }}\right)$, with $r_{\text {inj }}$ the injection radius. The solid-line histograms (in color) give the number of CRs in the local volume from each of the rings. The total number of CRs from all rings is shown in black, all as a function of CR age.

Figures 7/8 are similar to Figures 5/6, but for the SNR surface density defined by Green (2015) and Sasaki et al. (2004):

$$
N_{\mathrm{snr}}(r) \propto\left(\frac{r}{R_{\odot}}\right)^{\alpha} \exp \left(-\frac{r}{R_{\mathrm{snr}}}\right) .
$$

Here $\alpha=1.1, R_{\odot}=8.5 \mathrm{kpc}$ is the Galacto-centric radius of the Solar orbit around the Galactic Center and we take $R_{\mathrm{snr}}=5.4 \mathrm{kpc}$ in Figure 7 and $R_{\mathrm{snr}}=8 \mathrm{kpc}$ in Figure 8.

Figure 9 shows how the CRs inside the local volume around the Solar System are distributed over the accumulated grammage. Again we integrate over the entire simulation run. The contribution from each of the rings (solid colored lines) is shown 


\begin{tabular}{ccccc}
\hline SNRs & Distance $[\mathrm{kpc}]$ & Galactic longitude $(l)[$ degree $]$ & Age[kyr] & Position(X,Y,Z) $\mathbf{k p c}]$ \\
\hline \hline CTA1 & 1.40 & 119.5 & 24.5 & $(-9.18,1.20,0.28)$ \\
\hline G65.3+5.7 & 1.00 & 65.3 & 14 & $(-8.17,0.72,0.10)$ \\
\hline G73.9+0. 9 & 1.30 & 73.9 & 10 & $(-8.14,1.25,0.05)$ \\
\hline HB21 & 0.80 & 89.0 & 19 & $(-8.48,0.80,0.10)$ \\
\hline G114.3+0.3 & 0.70 & 114.3 & 41 & $(-8.79,0.64,0.03)$ \\
\hline HB9 & 1.00 & 160.9 & 7.7 & $(-9.44,0.33,0.08)$ \\
\hline S147 & 0.80 & 180.0 & 4.6 & $(-9.3,0.00,0.00)$ \\
\hline Vela & 0.30 & 263.9 & 11 & $(-8.53,-0.30,0.01)$ \\
\hline G299.2-2.9 & 0.50 & 299.2 & 5 & $(-8.26,-0.44,0.00)$ \\
\hline Cygnus Loop & 0.44 & 74.0 & 14 & $(-8.38,0.42,-0.03)$ \\
\hline \hline
\end{tabular}

Table 1. Parameters of known SNRs located within a distance of $1.5 \mathrm{kpc}$ from the Solar System considered in our calculation.

separately, as well as its sum (solid black line). The grammage distribution at the moment of escape for the Galaxy is shown in dashed lines. For the grammage calculation we assume the smooth density distribution of Eqn. (9). Simulation parameters are the same as before. The accumulated grammage becomes larger when the source is farther from the Sun. It also increases when the value of the ratio $\epsilon=D_{\perp} / D_{\|}$is small, because the CRs take longer to escape from the Galaxy, as discussed in some detail above.

\subsection{Results for CRs from four hypothetical sources at the same distance from Earth}

In order to investigate the effects of the strength of perpendicular diffusion on CR numbers at Earth we have simulated the propagation of CRs from four discrete equidistant sources (source distance: $5 \mathrm{kpc}$ from Earth). These sources are typically located on different sections of the JF spiral disk field, see Figure 10, bottom panel. It is assumed that the sources inject the CRs simultaneously at $t=0$. Again we perform simulations for two values of the ratio $\epsilon=D_{\perp} / D_{\|}=0.01$, 1 . The value of the parallel diffusion coefficient is kept constant at $D_{\|}=3 \times 10^{28} \mathrm{~cm}^{2} \mathrm{~s}^{-1}$. The thickness of the CR halo above the disk is taken to be $H_{\mathrm{cr}}=4 \mathrm{kpc}$. CRs escape freely once they reach $z= \pm H_{\mathrm{cr}}$. Corresponding values for CR ages in the local volume for other values of $D_{\|}$can be obtained from the scaling law $t \propto D_{\|}^{-1}$.

The top two panels in Figure 10 show, as a function of time, the normalized number of CRs inside the local volume for these four hypothetical sources. The black distribution shows the total number of CRs from all four sources. When $\epsilon=1$ (the case of isotropic diffusion) the distribution of the CRs over time from all four sources is identical, apart from statistical fluctuations due to the finite number of simulated CRs per source. Therefore, all four sources contribute equally to the local CR density at Earth, as expected for this case. The CRs also escape relatively quickly from the Galaxy.

In contrast: for $\epsilon=0.01$ the distribution over time becomes wider as the CRs take more time to escape from the Galaxy, as already discussed. By far the largest contribution now comes from Source 3 that lies on the same section of the JF spiral disk field as the Sun. CRs from that source can reach Earth relatively rapidly through parallel diffusion. The contribution from Sources 1 and 2 is reduced as a significant amount of cross-field diffusion is required, taking a longer time. There is no contribution from Source 4: CRs from this source have not enough time to diffuse the required distance across the magnetic field to reach the local volume before escaping from the Galaxy.

\subsection{Results for CRs from ten specific sources close to Earth}

Next we investigate the effect of the strength of perpendicular diffusion on the CR flux at Earth from ten specific nearby discrete sources. All sources lie within a distance of $1.5 \mathrm{kpc}$. We employ the same source set as the one employed by Thoudam (2007). Simulation parameters are the same as before.

For such nearby discrete sources, one expects significant temporal fluctuations in the local density of CRs at Earth. The exact position of these SNRs and the direction of the local Galactic magnetic field are shown in Figure 11. In order to simplify things we assume that each source produces the CRs impulsively at the time of the supernova explosion. In reality, most of the CRs are produced in a few Sedov-Taylor times $t_{\mathrm{ST}}$, given for typical SNR parameters by: 


$$
t_{\mathrm{ST}} \simeq \frac{R_{\mathrm{dec}}}{V_{\mathrm{f}}}=\frac{\left(3 M_{\mathrm{ej}} / 4 \pi \rho_{\mathrm{ism}}\right)^{1 / 3}}{\sqrt{2 E_{\mathrm{snr}} / M_{\mathrm{ej}}}} \simeq 1.7 \times 10^{3}\left(\frac{E_{\mathrm{snr}}}{10^{51} \mathrm{erg}}\right)^{-1 / 2}\left(\frac{n_{\mathrm{ism}}}{1 \mathrm{~cm}^{-3}}\right)^{-1 / 3}\left(\frac{M_{\mathrm{ej}}}{10 M_{\odot}}\right)^{5 / 6} \mathrm{yr} .
$$

Here $E_{\mathrm{snr}}$ is the mechanical energy driving the expansion of the supernova remnant, $M_{\mathrm{ej}}$ is the ejecta mass, $V_{\mathrm{f}}=$ $\sqrt{2 E_{\mathrm{snr}} / M_{\mathrm{ej}}}$ is the free-expansion velocity of the remnant for $t<t_{\mathrm{ST}}, R_{\mathrm{dec}}$ is the deceleration radius where the SNR has

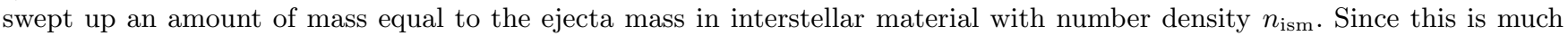
shorter than the typical $\left(10^{7} \mathrm{yr}\right) \mathrm{CR}$ residence time this simplification is justified.

In Figure 12 we show, for each of the sources of Table 1, the distribution of the normalized number of CRs that reside in the local volume around the Solar system as a function of time. Again we consider the two cases: $D_{\perp} / D_{\|}=1$ (upper plot), $D_{\perp} / D_{\|}=0.01$ (lower plot). The dashed distribution shows the total number of CRs from all sources. The associated grammage distribution is shown in Figure 13. The dashed distribution again is the total number of CRs from all ten SNRs sources. Again we choose $D_{\perp} / D_{\|}=1$ (upper plots), $D_{\perp} / D_{\|}=0.01$ (lower plots). Since these sources are relatively close, the difference between the case $D_{\perp} / D_{\|}=1$ and the case $D_{\perp} / D_{\|}=0.01$ is much less pronounced than in the other simulations presented here. Their location (shown in Figure 11) is such that for most of the sources the CRs can diffuse mostly along the local magnetic field to reach the Local Volume. The exceptions are S147 and HB9. In the case $D_{\perp} / D_{\|}=0.01$ they show the tell-tale sign of an absence of 'young' CRs in the lower panel of Figure 12, indicating that the CRs from these sources take longer to reach Earth when diffusion across the field is slow.

\subsection{Model test: the Boron to Carbon ratio}

The Boron to Carbon ratio B/C is widely used to determine key parameters (diffusion coefficient(s), scaling with CR energy etc.) of propagation models by comparing model predictions with observations. The Boron to Carbon ratio is (of many possible ratios of secondary to primary cosmic rays nuclei) the easiest to measure experimentally. Recently, this ratio has been determined with an accuracy of a few percent by the Alpha Magnetic Spectrometer (AMS-02) on the International Space Station ${ }^{1}$, over a relatively wide range of energies. Several other experiments like PAMELA ${ }^{2}$, and CREAM ${ }^{3}$ have also measured the $\mathrm{B} / \mathrm{C}$ ratio, in a range of kinetic energies between $100 \mathrm{MeV}$ and $1 \mathrm{TeV} /$ nucleon.

We have used the weighted slab method using the Grammage distributions for CR protons inside the local volume from the four rings to calculate B/C ratio as a function of kinetic energy per nucleon, using the path length distribution obtained from the simulations. In our calculation we have chosen the value of the parallel diffusion coefficient $D_{\|}$so that the B/C ratio obtained coincides with the observed value at $1 \mathrm{GeV} /$ nucleon. For its scaling with energy $\left(D_{\|} \propto E^{\delta}\right)$ we use a value of $\delta=0.33$, the value for standard Kolmogorov turbulence, which gives a reasonable fit to the observational data.

For these simulations this procedure is allowed since we have assumed that $\epsilon=D_{\perp} / D_{\|}$is constant. It should be pointed out that this assumption, although convenient, is often not justified since in many cases $D_{\|}$usually scales differently with CR energy than $D_{\perp}$. This was already discussed briefly in Section 2.2 of this paper. A non-trivial scaling of $\epsilon=D_{\perp} / D_{\|}$with CR energy introduces yet another parameter in the CR propagation model that has to be determined from theory and/or observations.

With the weighted slab method we calculate the Boron to Carbon abundance ratio as a function of energy per nucleon. As before we consider CRs in the local volume around the Solar System. In our calculation we assume that only Primary CRs nuclei $\mathrm{O}, \mathrm{N}$, and $\mathrm{C}$ serve as parent nuclei for B. The source abundance of each of the parent nuclei is taken from Strong \& Moskalenko (2001). Relevant nuclear data, such as cross sections, are found in Webber et al. (2003), Garcia-Munoz et al. (1987), and Ramaty et al. (1997). As before we consider primary CRs that originate from the four rings centered on the Galactic Center. Figure 14 shows the B/C abundance ratio, plotted as a function of energy per nucleon for two cases: isotropic diffusion with $D_{\perp}=D_{\|}$(Figure 14a) and strongly anisotropic diffusion with $D_{\perp}=0.01 D_{\|}$(Figure 14b). The observed ratio is shown for comparison. Figures $14 \mathrm{c}$ and $14 \mathrm{~d}$ show the total number of CRs in the local volume, adding the contributions from the four rings, for $\epsilon=1$ and for $\epsilon=0.01$, as indicated in each panel. One clearly sees a good agreement with observations. This in itself is not too surprising: in this cases the path-length distribution as a function of energy is set by the (identical) scaling of $D_{\|}$and $D_{\perp}$ with CR energy per nucleon. By choosing $D_{\|}$at $1 \mathrm{GeV} /$ nucleon in such a way that the calculated

\footnotetext{
1 http://www.ams02.org

2 https://www.ssdc.asi.it/pamela/

3 https://cosmicray.umd.edu/iss-cream/
} 
Boron to Carbon ratio matches the observed value hides the effect of a varying anisotropy in CR diffusion. We have adjusted the value of $D_{0}$ to produce the best fit with the observational data. We use $D_{0}=3 \times 10^{28} \mathrm{~cm}^{2} \mathrm{~s}^{-1}$ for strongly anisotropic diffusion with $\epsilon=D_{\perp} / D_{\|}=0.01$, and $D_{0}=2.8 \times 10^{27} \mathrm{~cm}^{2} \mathrm{~s}^{-1}$ for isotropic diffusion with $\epsilon=D_{\perp} / D_{\|}=1$. This reduction by an order of magnitude of $D_{0}$ in the latter case reflects the need to keep the typical residence time the same in order to fit the observations, see the related discussion in Section 3.1.

To illustrate this point: in Figure 15 we show the effect of varying the ratio $\epsilon=D_{\perp} / D_{\|}$, but now (unlike Figure 14) for a constant value of $D_{\|}=4 \times 10^{28} \mathrm{~cm}^{2} \mathrm{~s}^{-1}$. As expected the B/C ratio increases with decreasing $\epsilon$ : when $\epsilon$ is small (primary) CRs reside longer inside the Galaxy, thereby accumulating a larger grammage and producing more secondary CRs.

In our calculation we did not address the calculation of the B/C ratio in the energy below $1 \mathrm{GeV} /$ nucleon. It was found, see for instance Garcia-Munoz et al. (1987), and Webber \& Villa (2016), that the ratio increases with energy below $1 \mathrm{GeV} /$ nucleon, and decreases above this energy, instead of the monotonic decrease with energy expected in the simplest Galactic diffusion model. Below $1 \mathrm{GeV} /$ nucleon, the $\mathrm{B} / \mathrm{C}$ ratio may be influenced to a significant degree by the advection of CRs by a Galactic wind, see for instance Strong \& Moskalenko (1998), or by CR re-acceleration during the propagation through the Galaxy, see for instance Simon et al. (1986). Alternatively, this behavior can be explained by having a constant CR diffusion coefficient below a few $\mathrm{GeV} /$ nucleon.

\subsection{Model test: fit to the CR data}

The spectrum of CR nuclei appeared to be consistent with a single power law in particle rigidity as defined by Longair (2011) $R_{\mathrm{cr}}=p c / Z e, J_{\mathrm{cr}} \propto R_{\mathrm{cr}}^{-\delta}$ with slope $\delta \approx 2.7$ in the energy range from $\sim \mathrm{GeV}$ to few $\mathrm{PeV}$.

Recent experiments of (AMS-02) on the International Space Station, and PAMELA have measured the energy spectra of various elements of CRs from protons to heavier nuclei below $10^{6} \mathrm{GeV}$.

In Figure 16 we show the spectrum of Proton, Helium and Carbon nuclei $\times E^{2.7}$ as function of kinetic energy per nucleon for the case of the diffusion coefficient $D_{\perp} / D_{\|}=0.01$ compared by recent experiments of (AMS-02) on the International Space Station, and PAMELA, for proton: Aguilar et al. (2015), Adriani et al. (2013), for He: Aguilar et al. (2017), Adriani et al. (2011), for C: Aguilar et al. (2017), Adriani et al. (2014b). The best fit to the spectrum according to a power law is represented by the color solid line as indicated in the plot. One clearly sees a good agreement with observations.

Secondary CR nuclei are created by spallation of primary CRs that interact with matter during their journey through the Galaxy by means of nuclear interactions of heavier fragments with the ISM. Spallation processes will remove nuclei according to:

$$
\frac{\mathrm{d} N}{\mathrm{~d} \ell}=-\sigma_{\mathrm{sp}} n_{\mathrm{H}}(\boldsymbol{r}) N
$$

where $\mathrm{d} \ell=v \mathrm{~d} t$ is the path length increase in a time-span $\mathrm{d} t, n_{\mathrm{H}}(\boldsymbol{r})$ is the Hydrogen number density at CR position $\boldsymbol{r}$ and $\sigma_{\mathrm{sp}}$ is the total spallation cross section. The formal solution of this equation is:

$$
N(\ell)=N(0) \mathrm{e}^{-\lambda(\ell)}, \lambda(\ell) \equiv \frac{\sigma_{\mathrm{sp}} \Sigma_{\mathrm{cr}}(\ell)}{\mu m_{\mathrm{H}}}
$$

The total spallation cross sections are found, for instance in Longair $(2011) . \Sigma_{\mathrm{cr}}(\ell)$ is the accumulated grammage, $\mu \simeq 1.3$ is the mean mass per particle in units of the Hydrogen mass of the interstellar gas, and $m_{\mathrm{H}}$ is the the mass of the Hydrogen atom.

The survival probability is defined (from Eqn. 19) as:

$$
P(\ell) \equiv \mathrm{e}^{-\lambda(\ell)}
$$

In Figure 17 we show the survival probability $P(\ell)$ for five elements (Helium, Carbon, Oxygen, Silicon and Iron) as a function of grammage. The survival probability decreases for heavier nuclei due to an increase in spallation cross section with atomic mass number $A$ : roughly $\sigma_{\mathrm{sp}} \propto A^{2 / 3}$. 


\section{CONCLUSIONS}

In the model presented in this work we have studied the effects of anisotropic diffusion on the propagation and residence time of GCRs. We also considered the properties of the GCRs in a local volume around the Solar System. We considered CRs from sources (supernova remnants) with a smooth distribution of sources, located in different intervals in the Galacto-centric radius. We also considered the contribution from distinct sources, located at a fixed distance from the Sun, and from a set of specific, real-world sources within a distance of $1.5 \mathrm{kpc}$ from the Solar System. All simulations employed the same approach, based on the solution of stochastic differential equations, to describe the CR propagation in the Galaxy. This study of anisotropic diffusion has used the field model of Jansson and Farrar for the regular (large-scale) Galactic magnetic field.

Our simulations show that for a smooth distribution of CR sources (taken to be Type II supernova remnants) the distribution of CR age inside the local volume around the Solar System, and the residence time distribution at the moment of CR escape from the Galaxy, are fairly similar in shape when diffusion is isotropic $\left(D_{\perp}=D_{\|}\right)$. They become rather different when diffusion is strongly anisotropic $\left(D_{\perp}=0.01 D_{\|}\right)$. Because of the tightly-wound spiral geometry of the Jansson-Farrar field in the plane of the Galactic Disk CRs originating from Galacto-centric distances larger than the Solar distance $(\sim 8.5 \mathrm{kpc})$ are (relatively speaking) depressed in the case of anisotropic diffusion. They must diffuse a significant distance across the large-scale magnetic field, which takes longer for small $D_{\perp}$, allowing a larger fraction to escape from the Galaxy before reaching the local volume. Given the field geometry this effect is not completely compensated by the larger residence time for the CRs in this case.

In addition we considered CRs that originate from ten discrete sources with known age and position inside the local volume, listed in Table 1. For this case we find the following: for somewhat distant sources (distance: $5 \mathrm{kpc}$, comparable to the assumed thickness $H_{\mathrm{cr}}=4 \mathrm{kpc}$ of the CR halo) the effect of anisotropic diffusion on the distribution of CR age and accumulated grammage inside the local volume is still visible, but not as pronounced as in the case where we consider CRs originating from the whole Galactic disk. For the subset of nearby sources (sources with distance less than $1.5 \mathrm{kpc}$ ) the differences between the case of isotropic diffusion and anisotropic diffusion are relatively small. If diffusion is very anisotropic $(\epsilon=0.01)$ sources not connected to the local volume by a field line of the Jansson-Farrar field near the disk mid-plane field contribute little to the local CR flux.

We have shown that for a constant (energy-independent) value of $\epsilon=D_{\perp} / D_{\|}$our code reproduces to observed Boron to Carbon ratio provided one normalizes $D_{\|}$in such a way that the value of $\mathrm{B} / \mathrm{C}$ at $1 \mathrm{GeV} /$ nucleon matches the observed value. This conclusion will change as soon as one allows $D_{\perp}$ and $D_{\|}$to scale differently with CR energy/nucleon.

The results of this paper demonstrate that it is relatively simple to simulate anisotropic CR diffusion in a large-scale magnetic field. Of course, a more realistic model will have to include additional physical effects that are now commonly included in CR propagation codes. In what follows we mention a few of these effects.

First of all we have neglected any CR energy changes during propagation. It is often assumed, see for instance that CRs are re-accelerated after leaving their source, either by stochastic acceleration in interstellar hydromagnetic turbulence (Fermi-II acceleration) or during random encounters with expanding supernova remnants. It has been suggested by Drury \& Strong (2017) that as much as $50 \%$ of the power needed to maintain the Galactic CR population against losses is provided by re-acceleration during propagation through the ISM. In either case the evolution of the momentum distribution $f(p)=$ $\mathrm{d} N /\left(4 \pi p^{2} \mathrm{~d} p\right)$ can be described by a diffusion equation in momentum space of the type:

$$
\left(\frac{\partial f}{\partial t}\right)_{\mathrm{acc}}=\frac{1}{p^{2}} \frac{\partial}{\partial p}\left(p^{2} D_{p} \frac{\partial f}{\partial p}\right) .
$$

Here $D_{p}$ is the momentum diffusion coefficient. Re-acceleration is easily incorporated into codes such as this by adding CR momentum as an additional dimension (in effect working in phase space) and integrating the following Itô equation for CR momentum in addition to Eqn. (2):

$$
\Delta p=\left\langle\frac{\mathrm{d} p}{\mathrm{~d} t}\right\rangle \Delta t+\sqrt{2 D_{p} \Delta t} \xi_{p}
$$

Here

$$
\left\langle\frac{\mathrm{d} p}{\mathrm{~d} t}\right\rangle \equiv \frac{1}{p^{2}} \frac{\partial}{\partial p}\left(p^{2} D_{p}\right)
$$


is the mean momentum gain due to the Fermi-II process and $\xi_{p}$ is another random variable, with zero mean and unit variance. The effect of energy losses (ionization losses, expansion losses etc.) are easily added to the regular momentum gain term, the first term on the right-hand side of Eqn. (22).

In the second place: we have employed the simplest possible model, where $D_{\perp} / D_{\|}$is a constant and independent of CR energy. The precise form of perpendicular diffusion, determining the scaling of $D_{\perp}$ with CR energy and its relation to $D_{\|}$, is a complicated problem with a range of possible regimes and scalings.

Finally: this model is (by design) a test-particle model. This makes it difficult to incorporate the effect of scattering of CRs by self-generated hydromagnetic waves if wave generation depends on the details of the CR energy distribution or the CR number density.

In a follow-up to this research we will consider two of the effects also neglected in these simulations: the influence of a Galactic wind and drift of CRs in the magnetic field. 

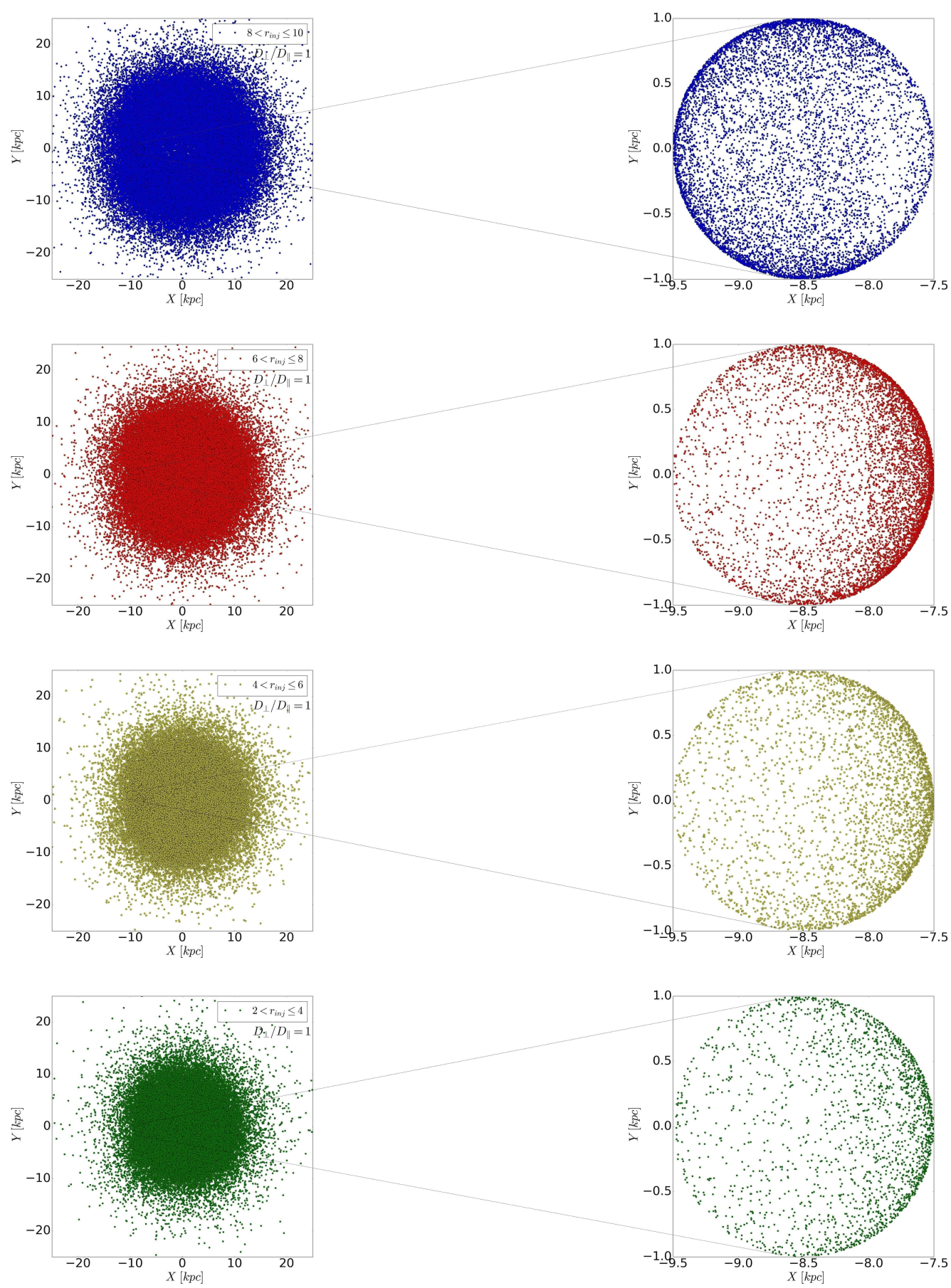

Figure 1. This figure shows the position of diffusing CRs in the plane of the disk at the moment they reach the upper or lower boundary at $z= \pm H_{\mathrm{cr}}=4 \mathrm{kpc}$ or $r=r_{\max }=20 \mathrm{kpc}$, for each rings as indicated in each panel. All particles are injected uniformly and randomly in each ring (left column). The right column shows the projected density of CRs inside the local volume around the Solar System for each of the rings. The asymmetry in the distribution reflects the position of the Sun with respect to the rings and shows the general radial gradient in the $\mathrm{CR}$ density. Here we assume isotropic diffusion $\left(D_{\perp} / D_{\|}=1\right)$ so that there is no influence of the large-scale (regular) Galactic magnetic field. 

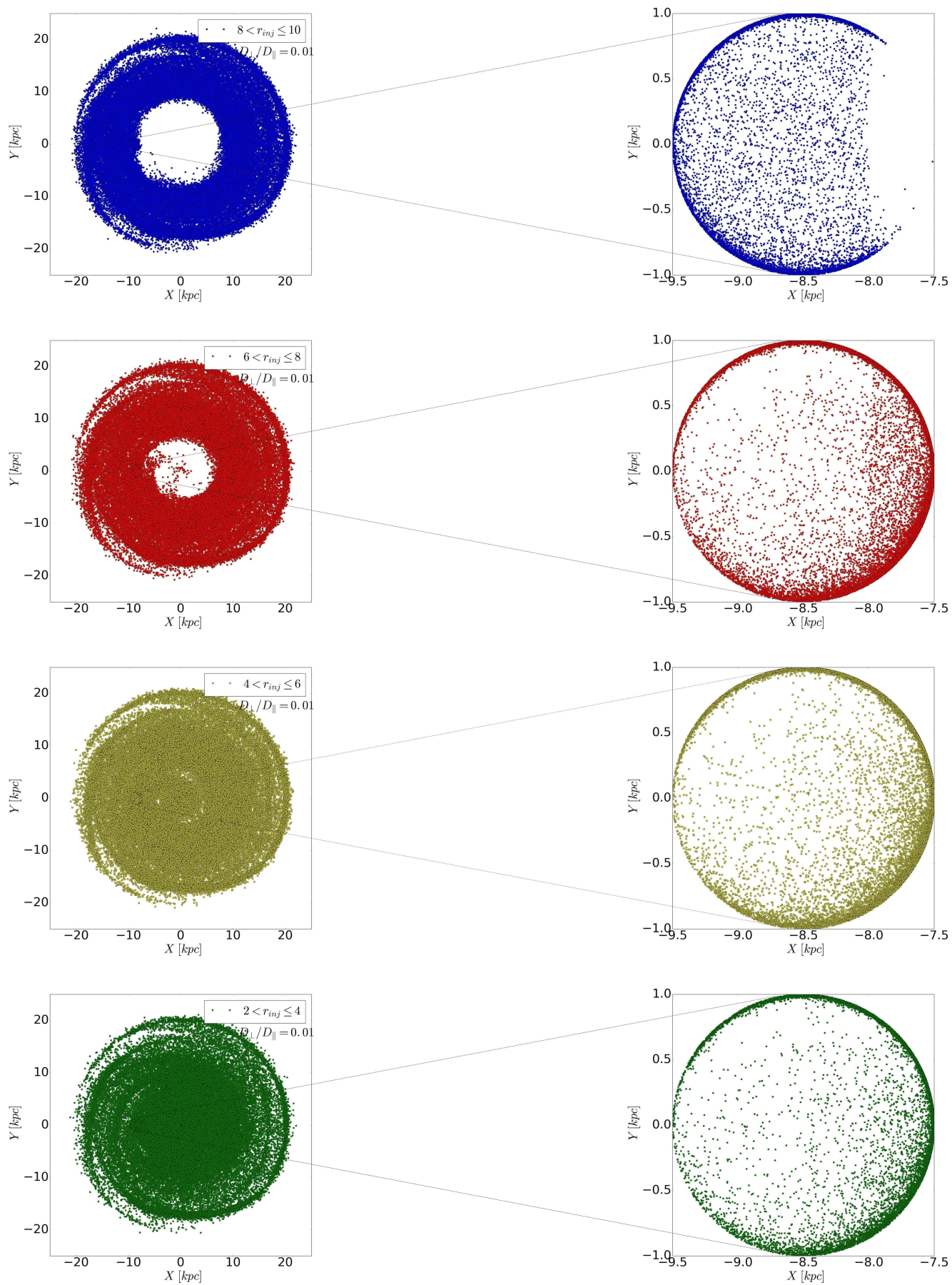

Figure 2. As in figure 1, but now for strongly anisotropic diffusion with the value of the ratio $D_{\perp} / D_{\|}=0.01$. One now sees the spiral structure of the disk field in the CR distribution, as CRs tend to follow magnetic field lines. 

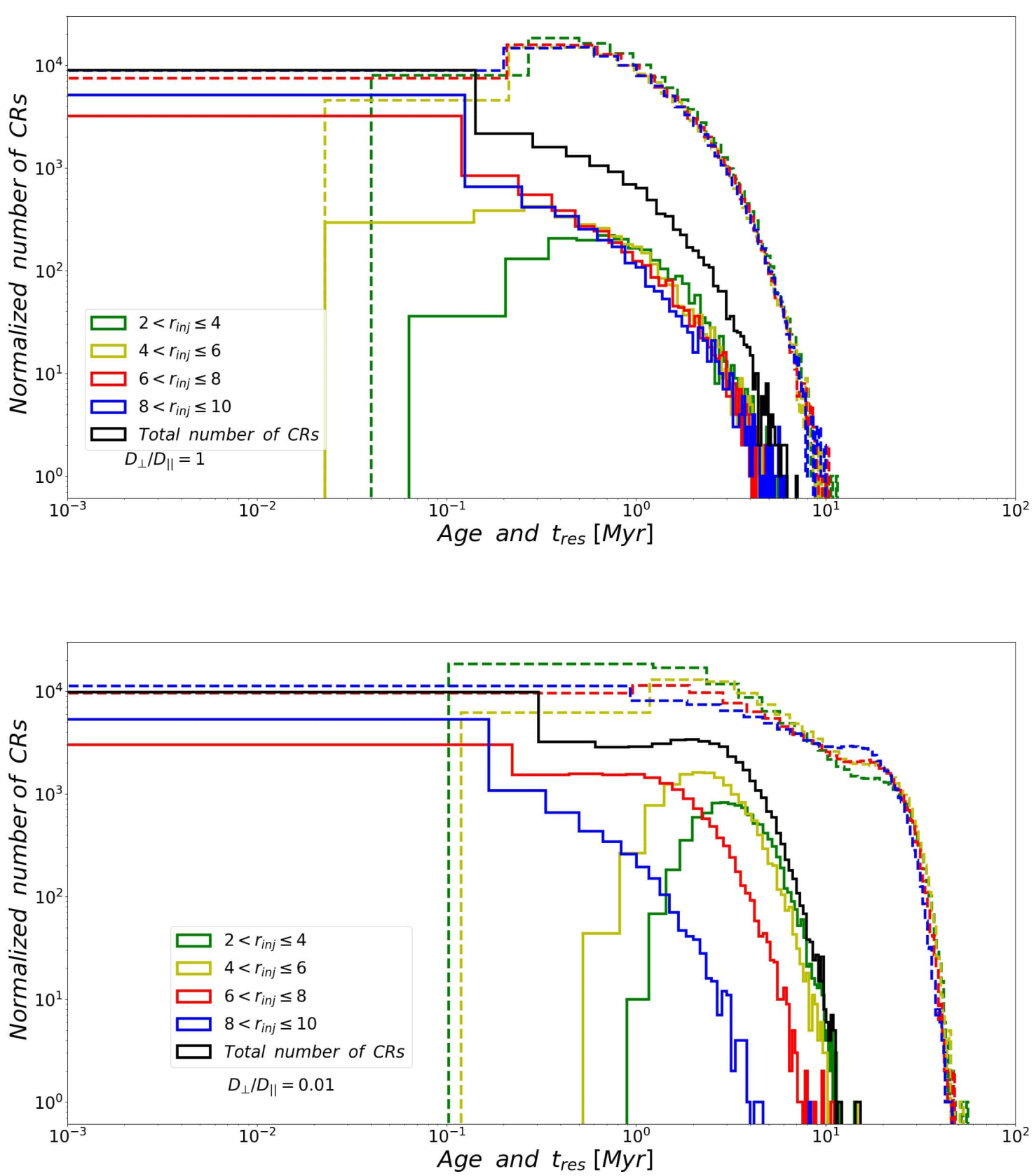

Figure 3. This figure shows, for each ring, the normalized distribution over age of the CRs observed in the local volume around the Solar System (solid lines), as well as the normalized distribution of the total residence time in the Galaxy (dashed lines). The black solid line shows the total number of CRs. All particles are injected randomly in the four rings with a uniform distribution for the value of the ratio $D_{\perp} / D_{\|}=1$ (upper panel: isotropic diffusion) and $D_{\perp} / D_{\|}=0.01$ (lower panel: anisotropic diffusion). 

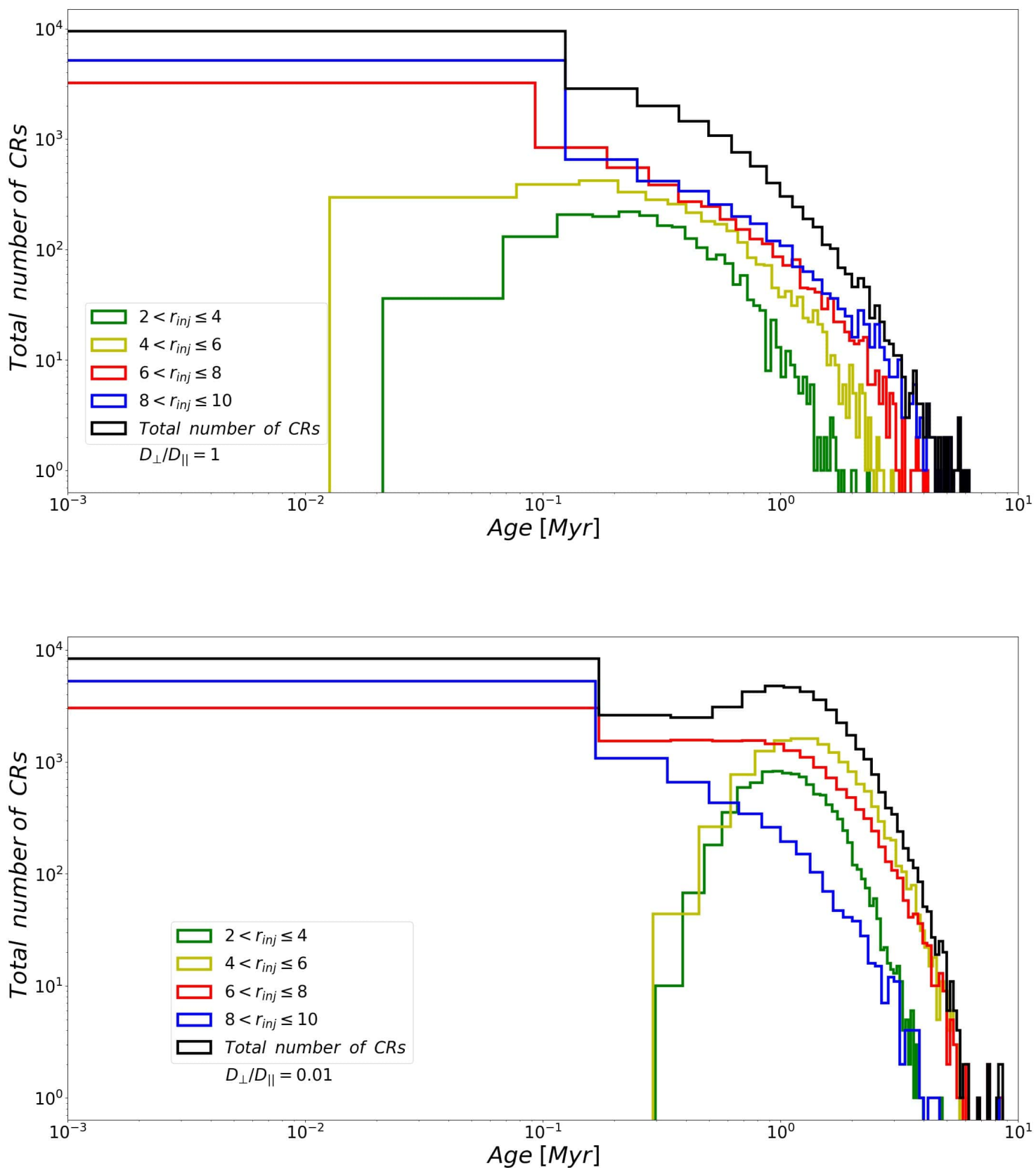

Figure 4. This figure shows, for each of four rings, the age distribution of the total number of CRs per unit disk area. The black solid line shows the total CRs come from all rings together. In each of the four rings CRs are injected randomly with a uniform distribution of sources (Type II supernova remnants) over disk area. Results are shown for $D_{\perp} / D_{\|}=1$ (upper panel: isotropic diffusion) and for $D_{\perp} / D_{\|}=0.01$ (lower panel: anisotropic diffusion). Note that in the latter case for the two inner rings $\left(r_{\text {inj }} \leq 6 \mathrm{kpc}\right)$ there are no CRs reaching the observer with a small age: the slow diffusion across the field prevents this. 

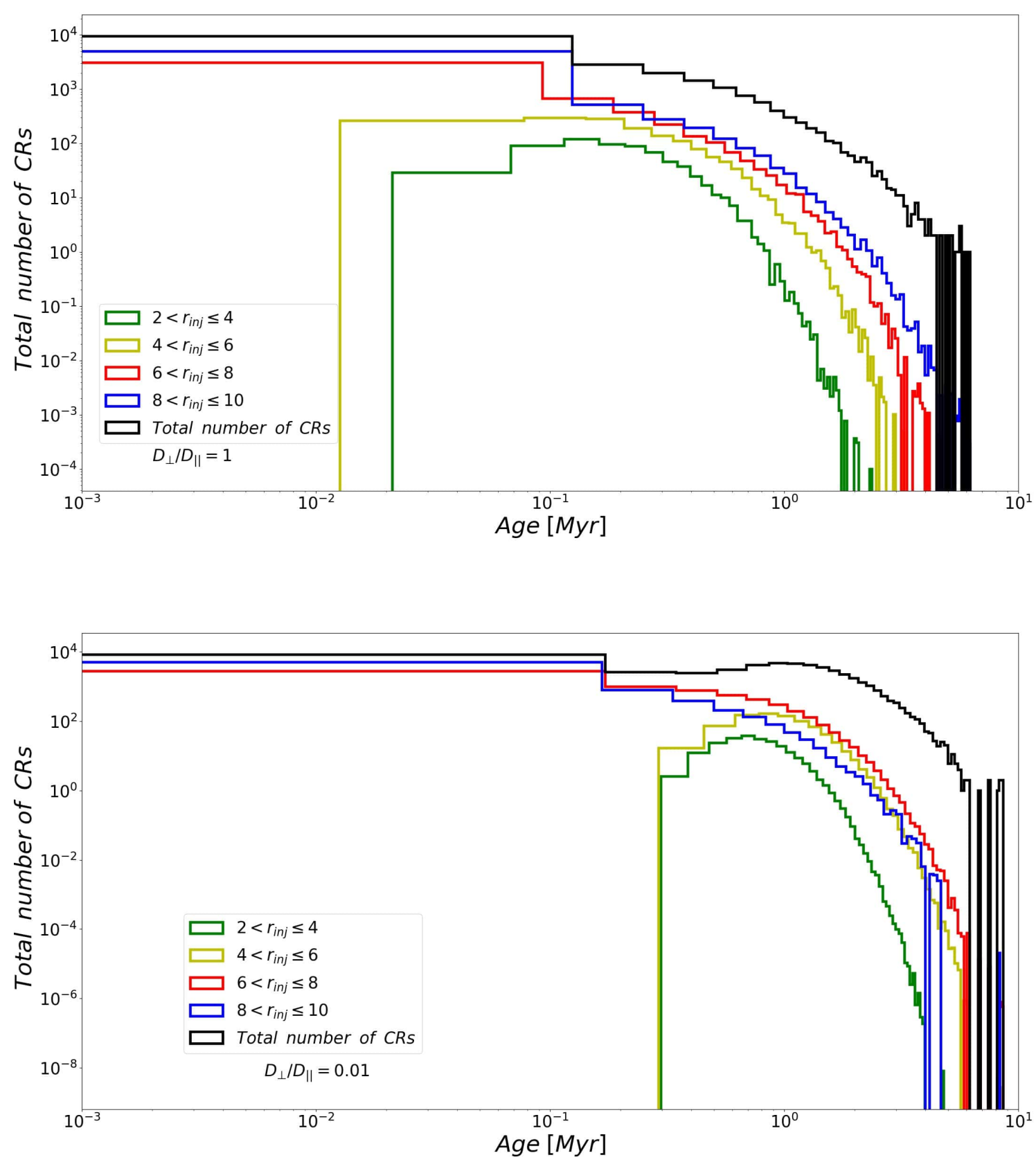

Figure 5. As in figure 4, but using the exponential surface density distribution (15) for the number of Type II supernova remnants. In this figure we employ a value of $R_{\mathrm{snr}}=5.4 \mathrm{kpc}$. 

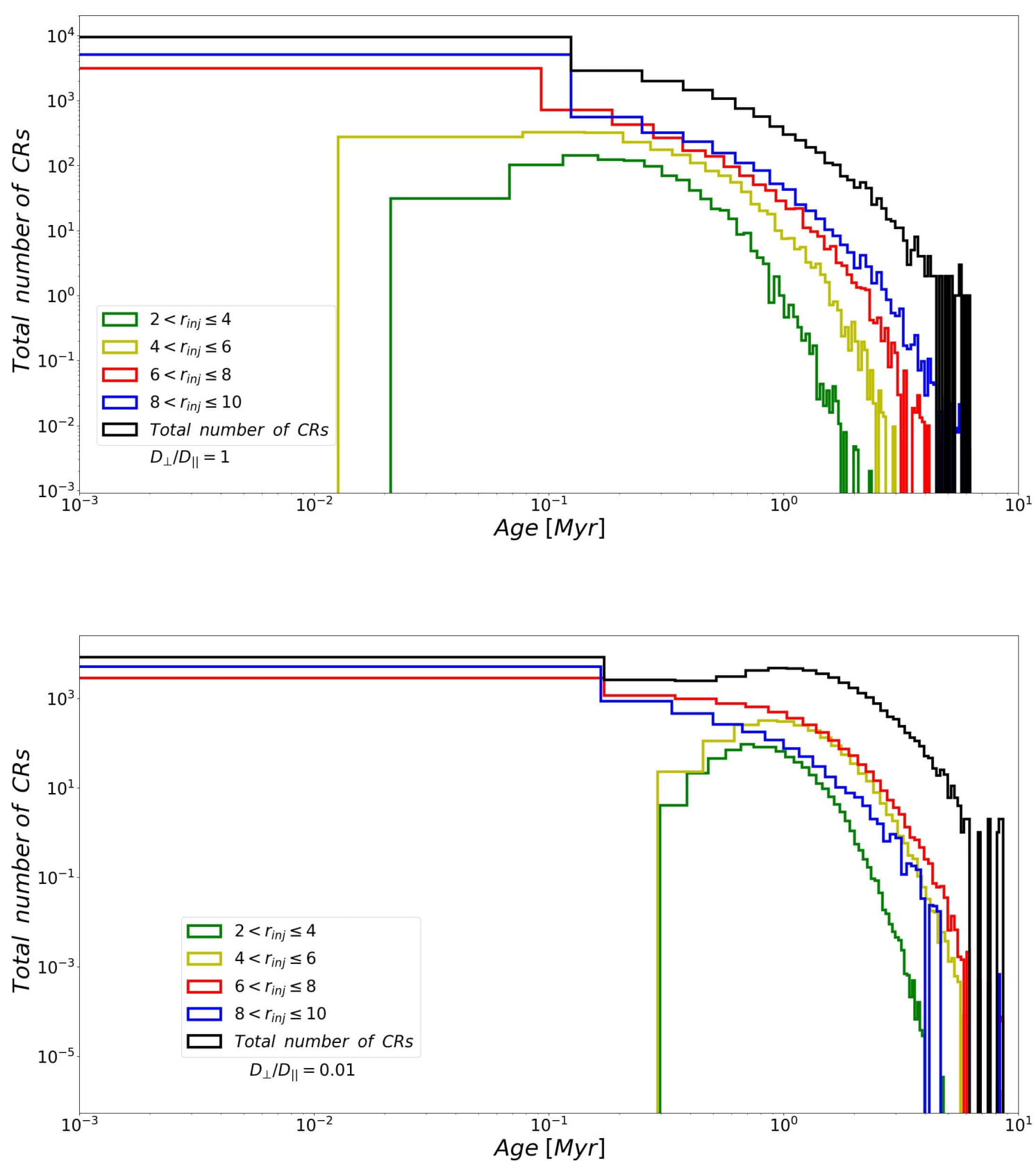

Figure 6. As in figure 4 , but now for the value of $R_{\mathrm{snr}}=8 \mathrm{kpc}$. 

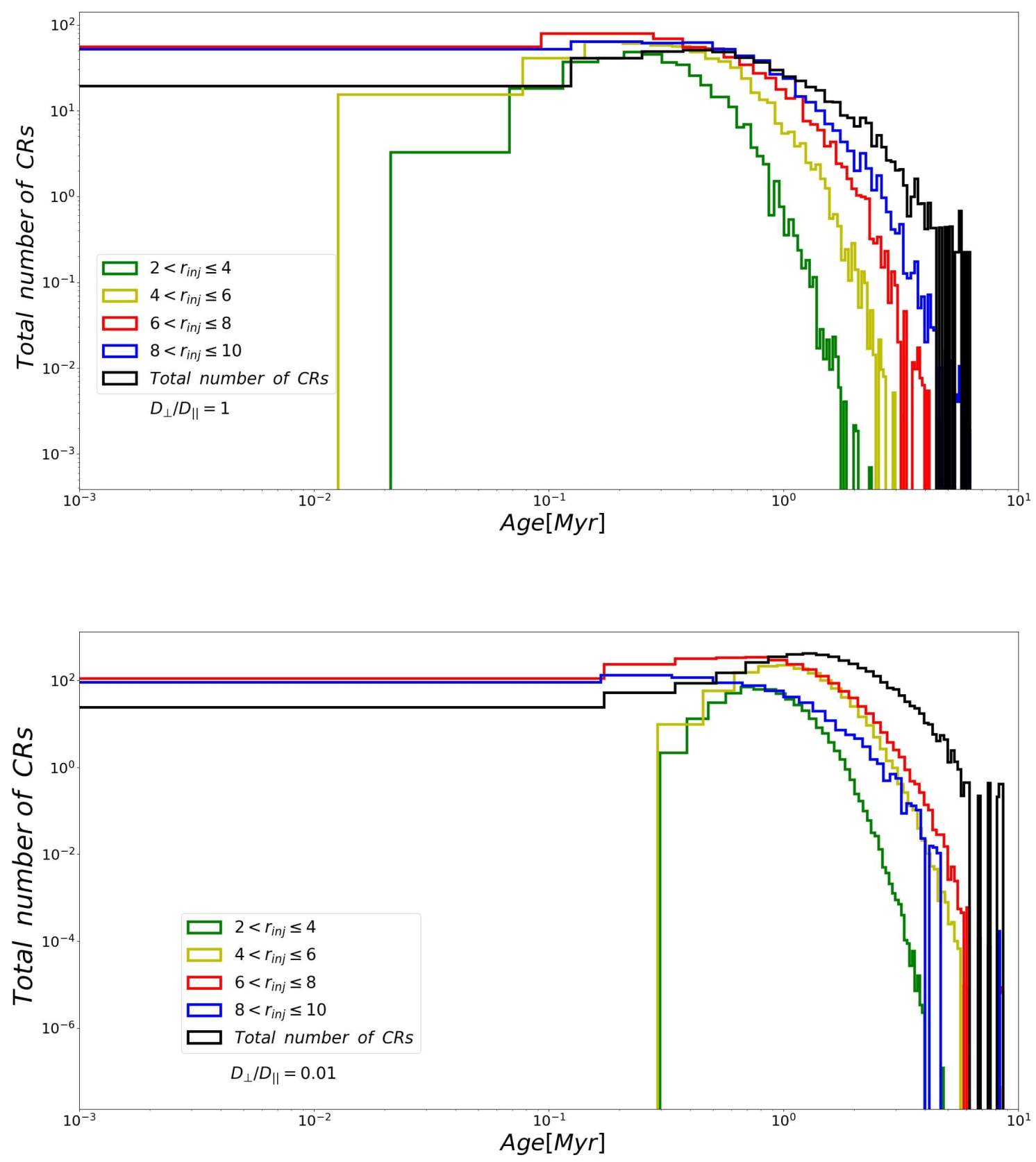

Figure 7. As in figure 4 , now using the supernova surface density distribution (16) with $R_{\mathrm{snr}}=5.4 \mathrm{kpc}$. 

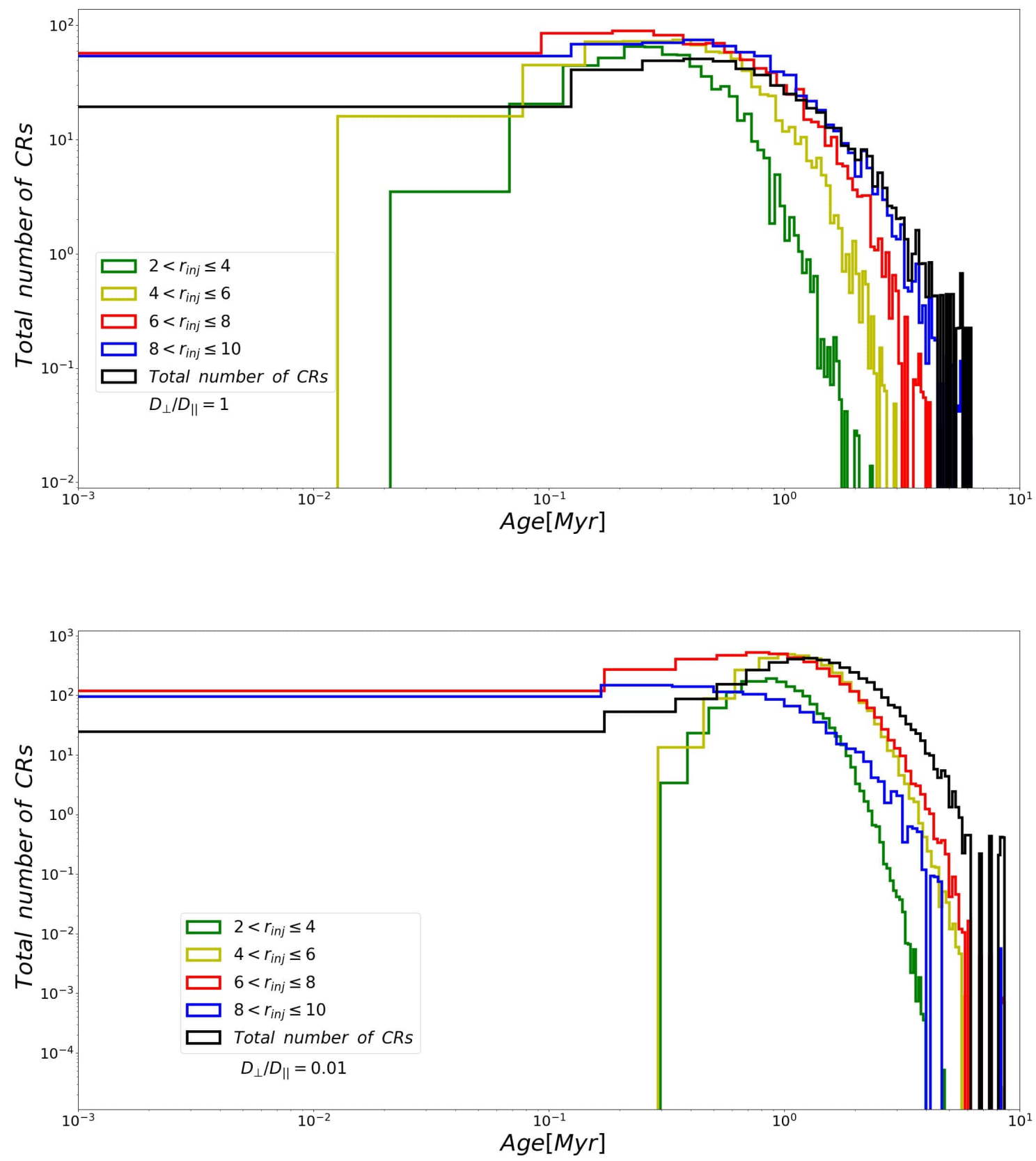

Figure 8. As in figure 8, for a value of $R_{\mathrm{snr}}=8 \mathrm{kpc}$. 

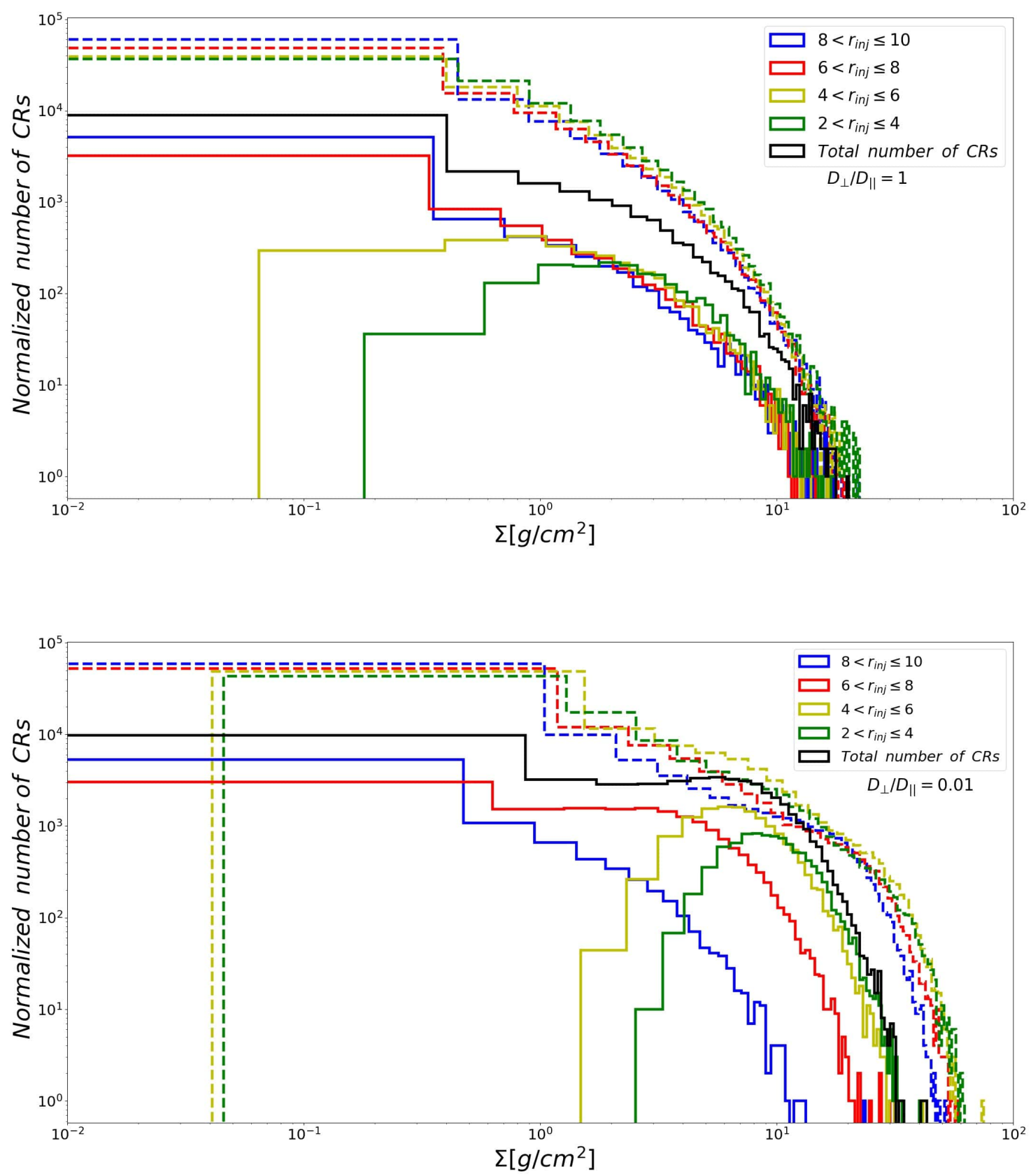

Figure 9. The normalized grammage distribution for the CRs observed in the local volume around the Solar System (solid lines). The black solid line shows the same for all CRs. The dashed lines show the same, but now at the moment of escape when the CRs reach the edge of the CR halo at $|z|=H_{\text {cr }}$ with $H_{\text {cr }}=4 \mathrm{kpc}$. The two cases shown are $D_{\perp} / D_{\|}=1$ (upper panel: isotropic diffusion) and $D_{\perp} / D_{\|}=0.01$ (lower panel: anisotropic diffusion). 

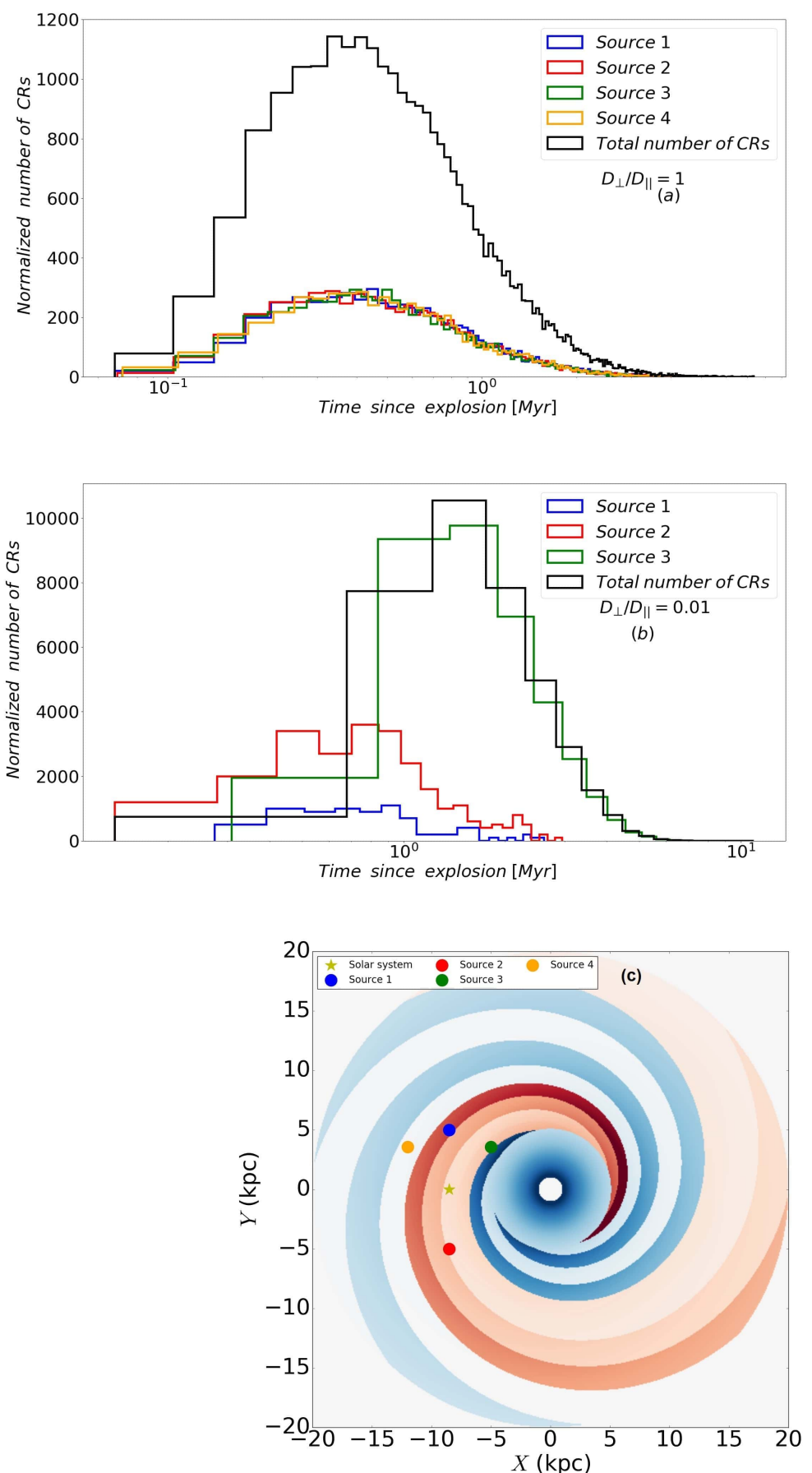

Figure 10. This figure shows the CR proton flux at the position of the Sun due to a set of four hypothetical single sources. These sources are located at a distance of $5 \mathrm{kpc}$ in different sectors of the Galactic magnetic field. The sources marked as $(1,2,3,4)$. The solid black line shows the total CRs come from all four sources. For the value of the ratio $D_{\perp} / D_{\|}=1$ (a) $D_{\perp} / D_{\|}=0.01$ (b), (c) shows the position of the discrete sources (circles) in the Galactic magnetic field. Yellow (star) indicates the position of our Solar System. 


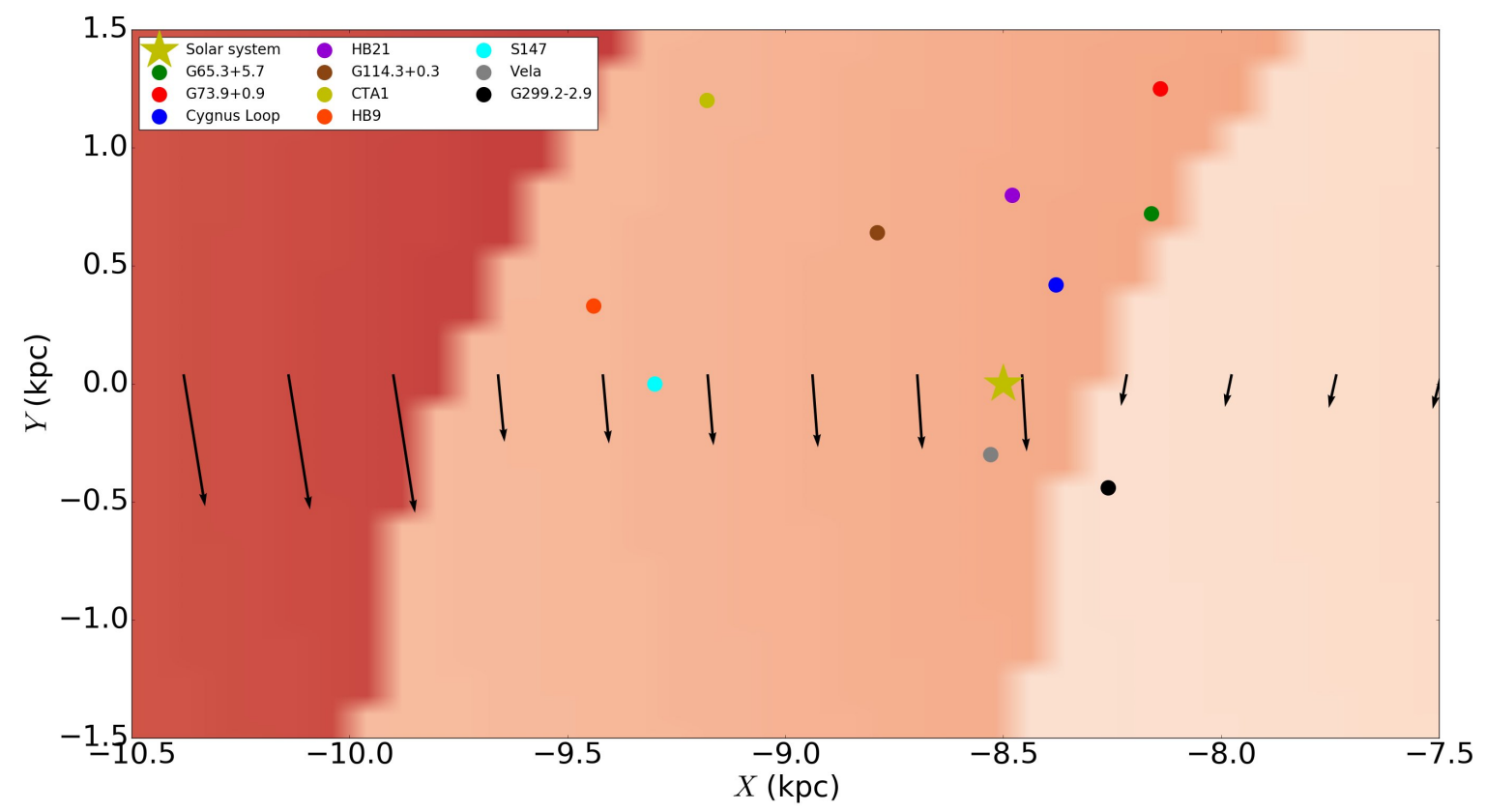

Figure 11. This figure shows the position of the known nearby SNRs (circles) in the Galactic magnetic field. The yellow star indicates the position of our Solar System. The arrows indicate the local direction of the Galactic magnetic field, projected onto the plane of the Galactic disk. 

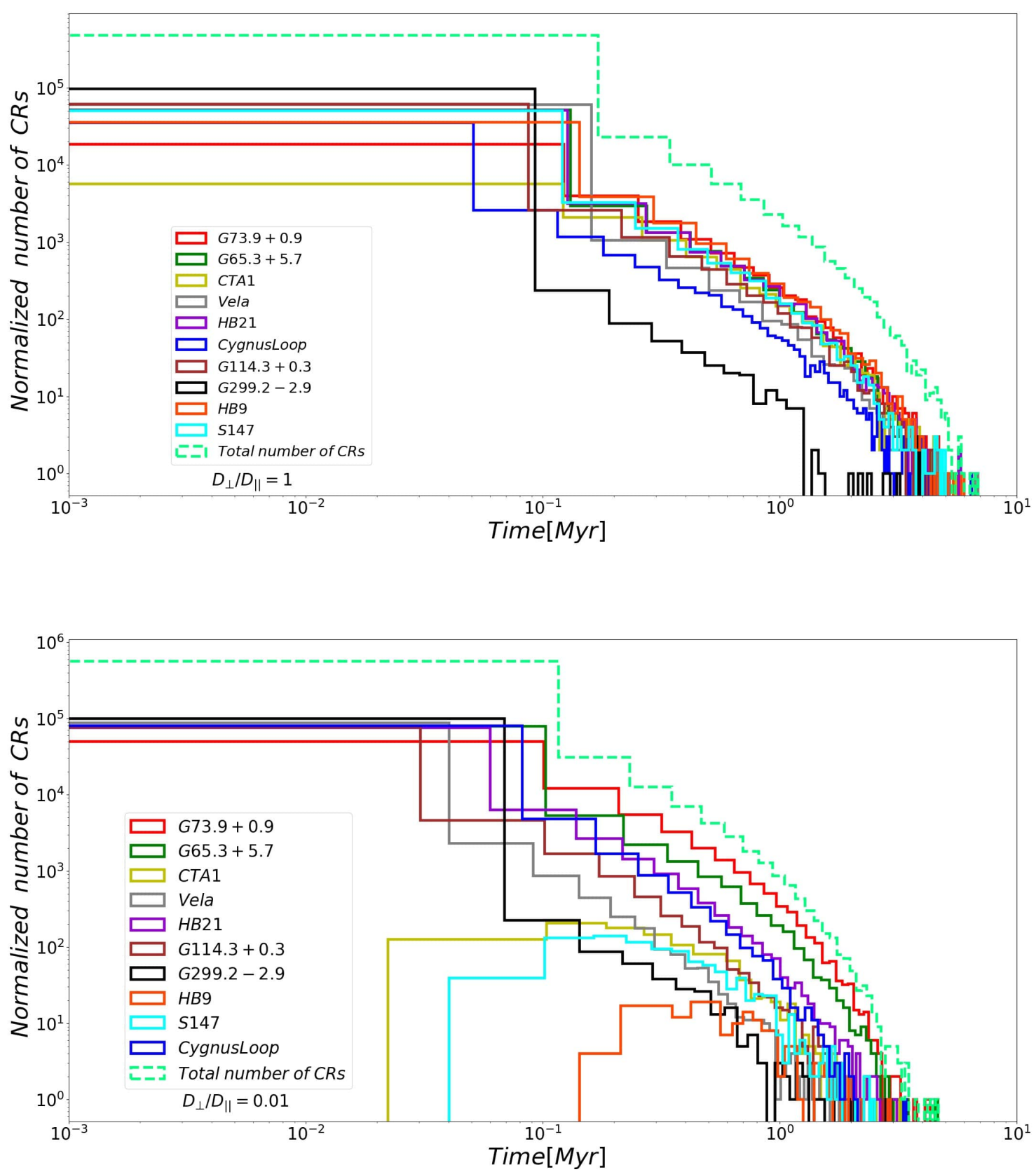

Figure 12. This figure shows, as a function of time, the normalized number of CRs that reside within $0.5 \mathrm{kpc}$ of the Solar System. The sources are the SNRs listed in table 1. The dashed line shows the total number of CRs from all ten sources together. Again we show the results for $D_{\perp} / D_{\|}=1$ (upper panel) and $D_{\perp} / D_{\|}=0.01$ (lower panel). 

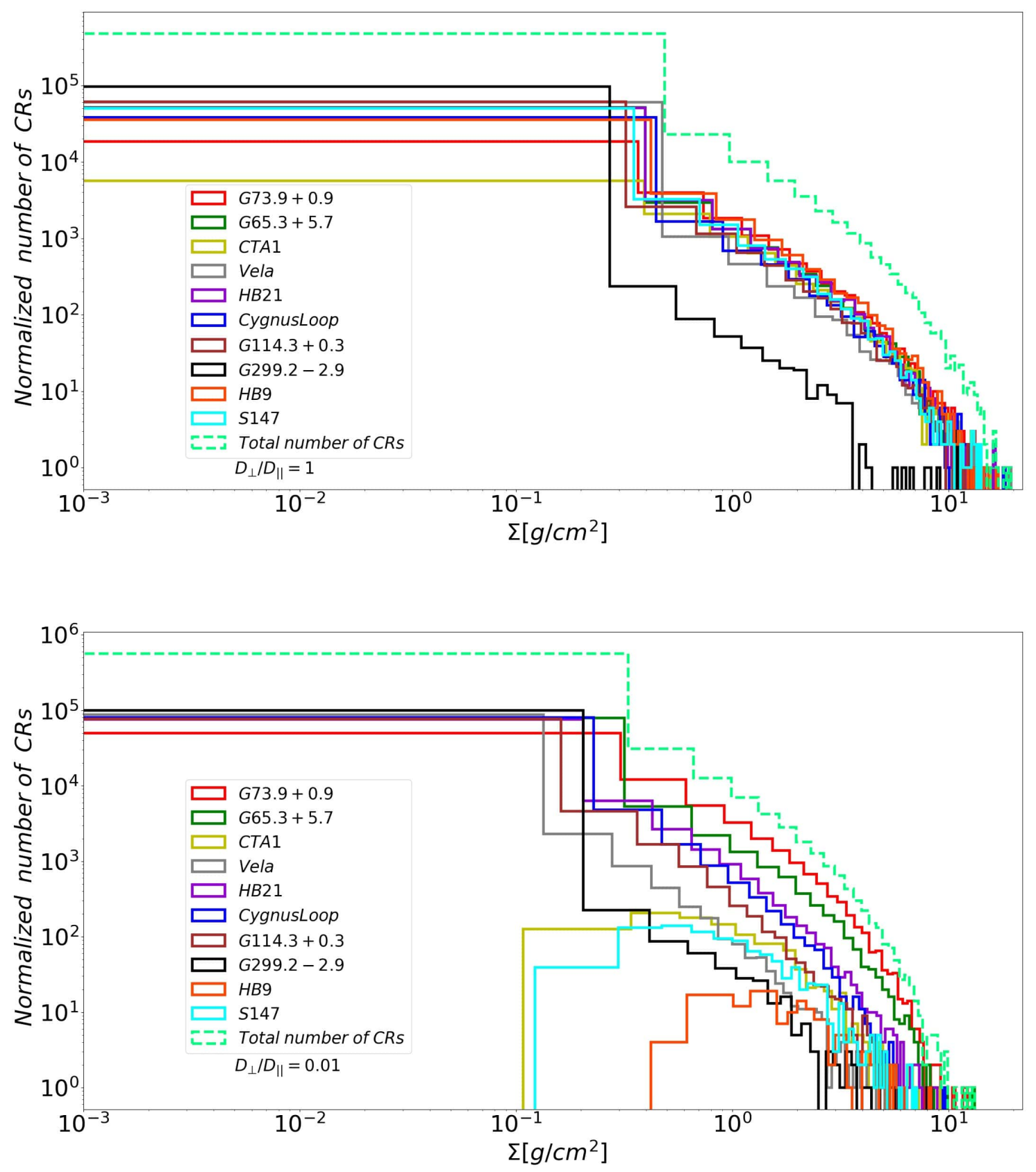

Figure 13. As in figure 12, but now showing the distribution over grammage. 

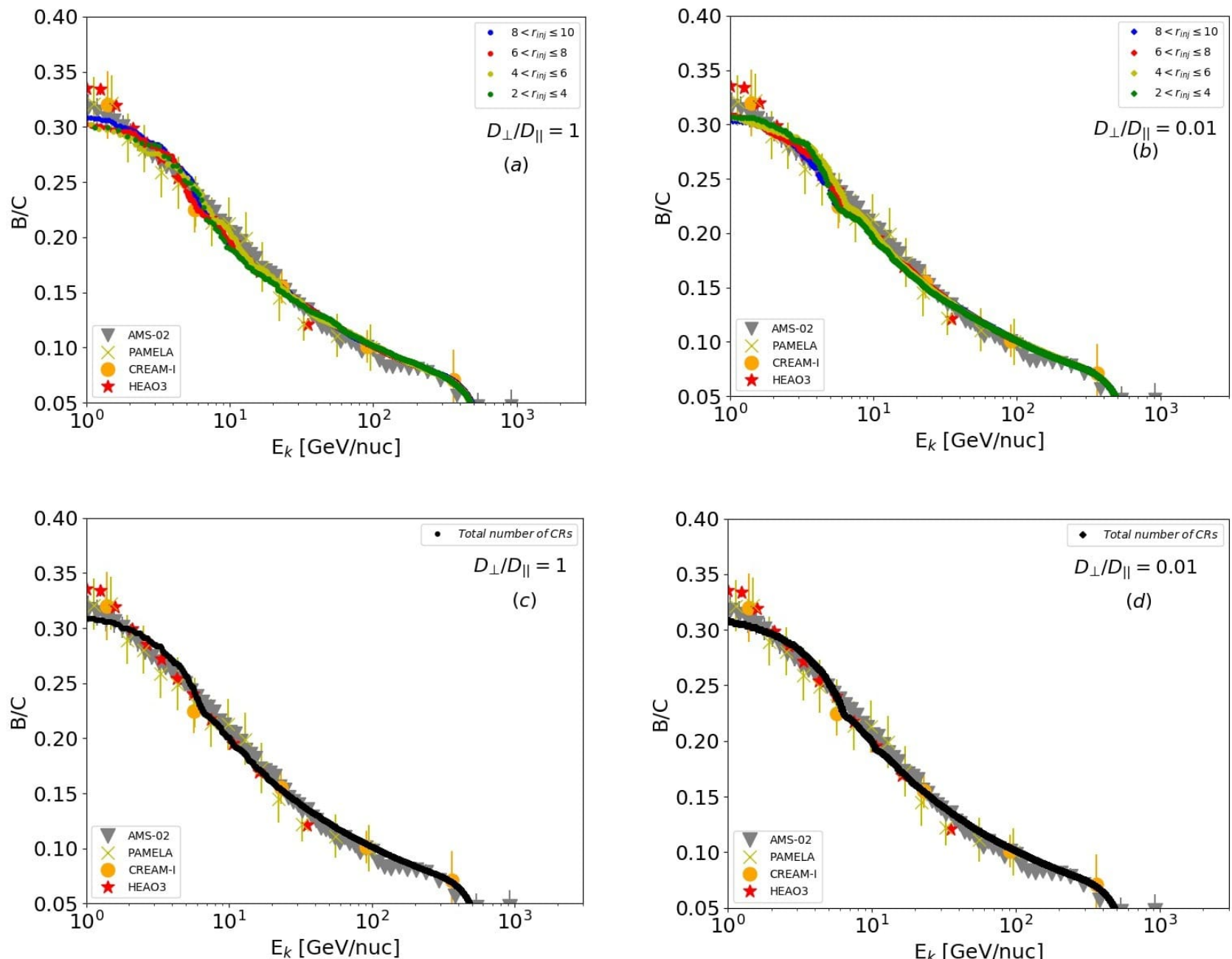

Figure 14. This figure shows the Boron- Carbon abundance ratio $(\mathrm{B} / \mathrm{C})$ as a function of kinetic energy per nucleon for two cases of the diffusion coefficient $D_{\perp} / D_{\|}=1(a), 0.01(b)$ for the four rings in the local volume around the Solar System (solid lines), compared to observational data from HEAO3 Engelmann et al. (1990), CREAM Ahn et al. (2008), AMS-02 Aguilar et al. (2016), and PAMELA Adriani et al. (2014a). The black solid line is the total number of CRs come from all of the four rings, as indicated in each panel. We use the value of $D_{0}$ to produce the best fit with the observational data. We use $D_{0}=3 \times 10^{28} \mathrm{~cm}^{2} \mathrm{~s}^{-1}$ for $D_{\perp} / D_{\|}=0.01$ (anisotropic diffusion), and $D_{0}=2.8 \times 10^{27} \mathrm{~cm}^{2} \mathrm{~s}^{-1}$ for $D_{\perp} / D_{\|}=1$ ( isotropic diffusion). 


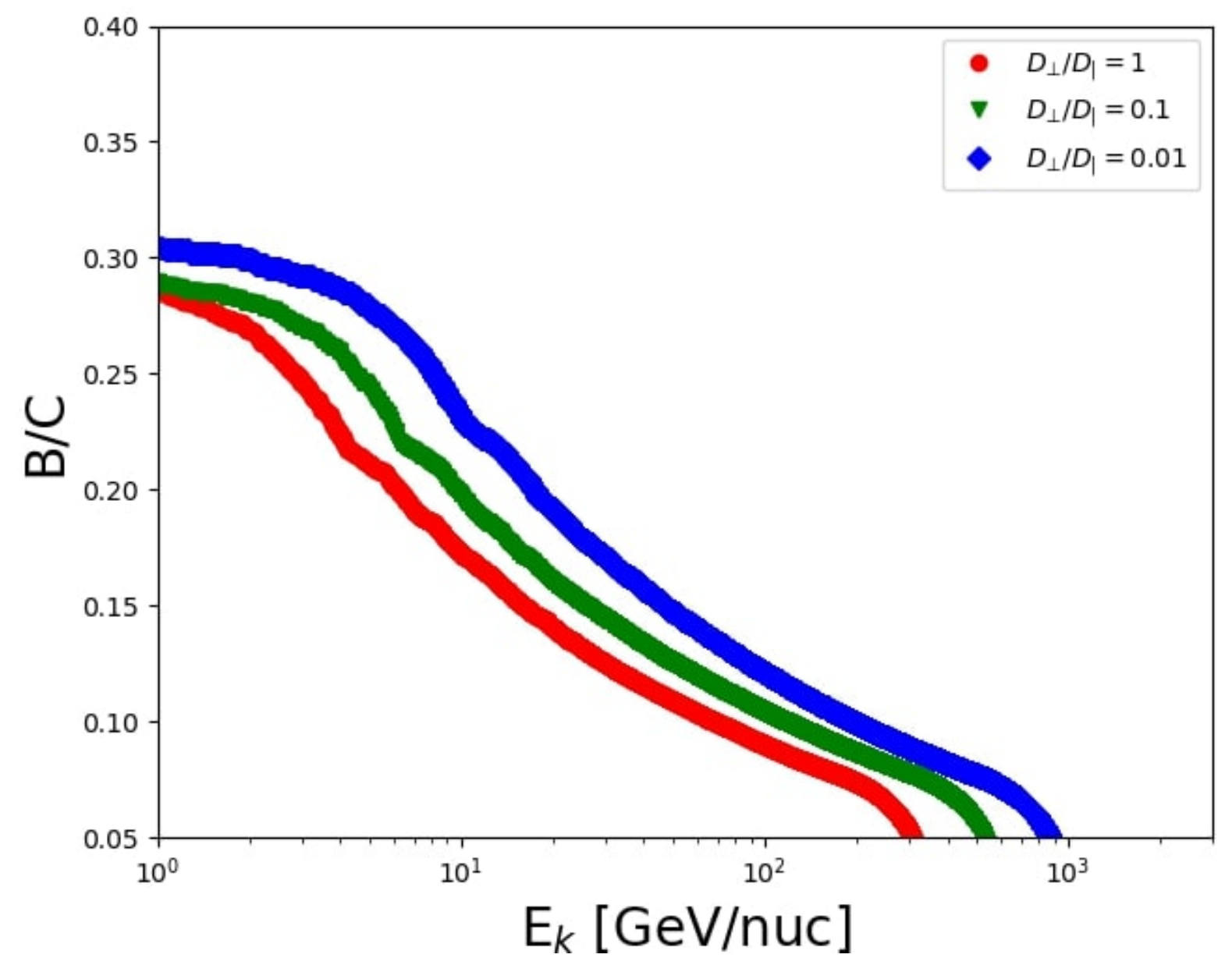

Figure 15. This figure shows the Boron- Carbon abundance ratio (B/C) as a function of kinetic energy per nucleon for the value of the ratio $D_{\perp} / D_{\|}=0.01,0.1,1$. In contrast to what is shown in Figure 14 the value of $D_{\|}$is now fixed at $D_{\|}=4 \times 10^{28} \mathrm{~cm}^{2} \mathrm{~s}^{-1}$. 


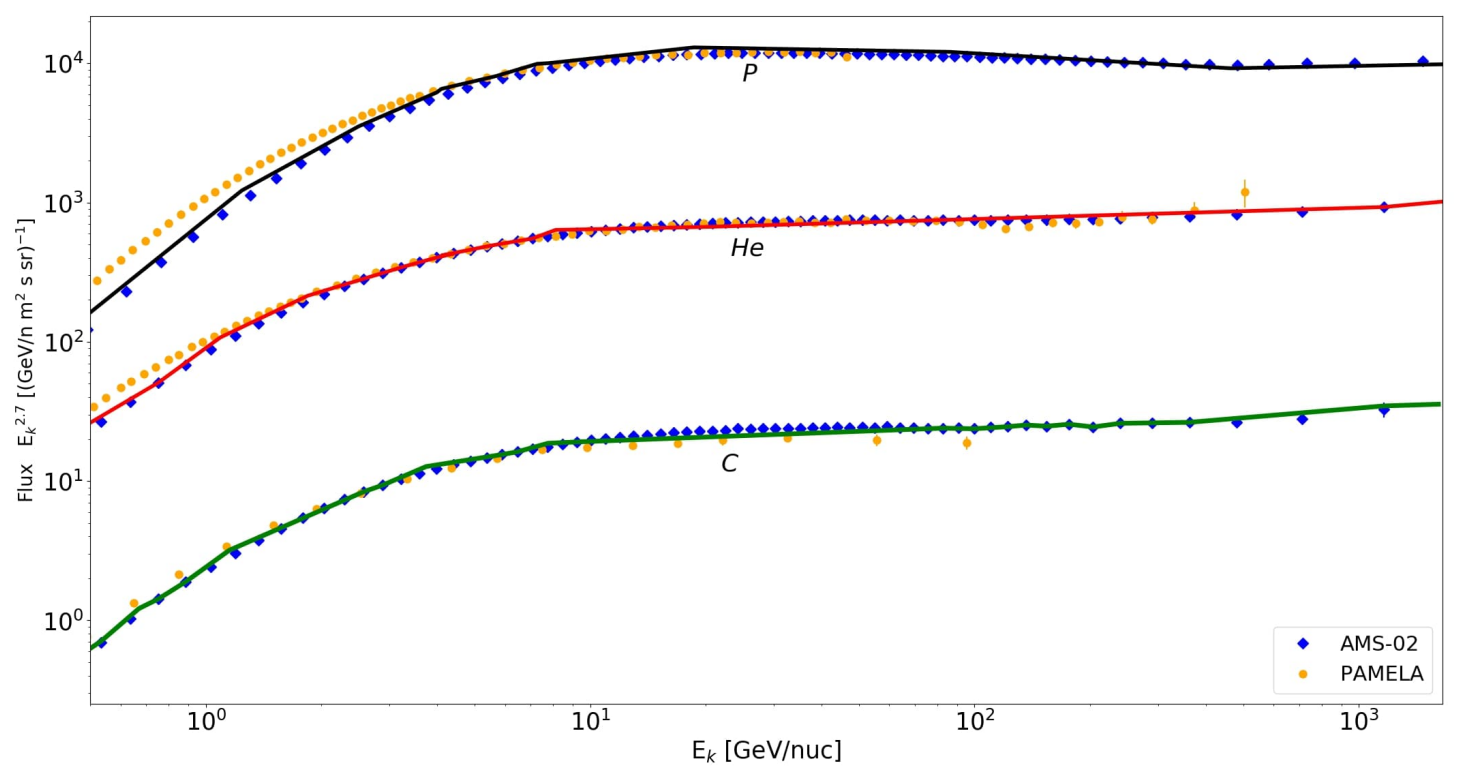

Figure 16. This figure shows The spectrum of Proton, Helium and Carbon as function of kinetic energy per nucleon for the case of the diffusion coefficient $D_{\perp} / D_{\|}=0.01$, compared to observational data from for Proton: Aguilar et al. (2015) ,Adriani et al. (2013), for He: Aguilar et al. (2017), Adriani et al. (2011), for C: Aguilar et al. (2017), Adriani et al. (2014b). 


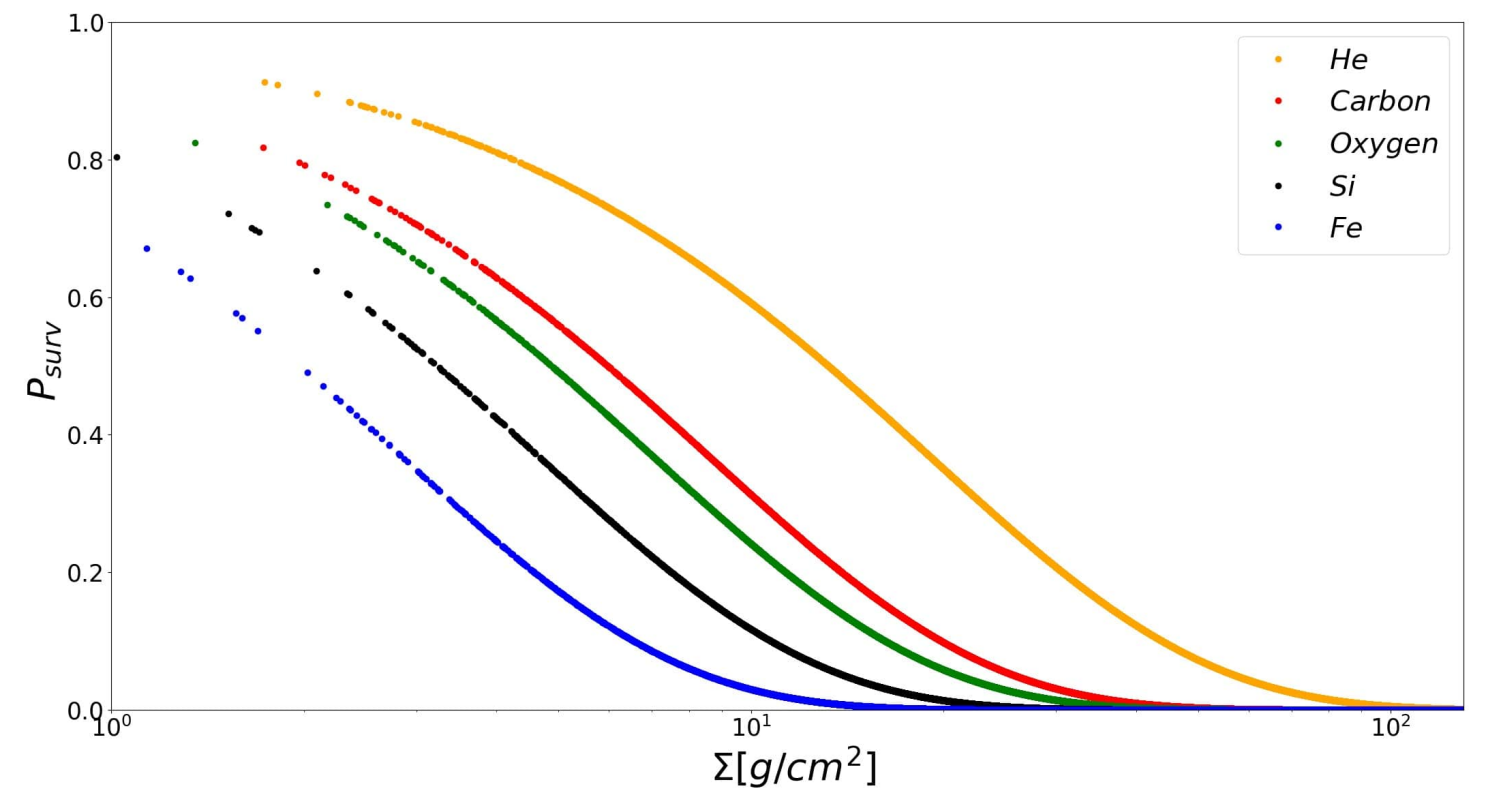

Figure 17. This figure shows the survival probability for some CR nuclei as a function of grammage.

\section{REFERENCES}

AL-Zetoun A., Achterberg A., 2018, MNRAS,

Abramowitz M., Stegun I. A., 1972, Handbook of Mathematical Functions, ninth printing. Dover Publications Inc., New York, USA

Adriani O., et al., 2011, Science, 332, 69

Adriani O., et al., 2013, The Astrophysical Journal, 765, 91

Adriani O., et al., 2014a, ApJ, 791, 93

Adriani O., et al., 2014b, The Astrophysical Journal, 791, 93

Aguilar M., et al., 2015, Phys. Rev. Lett., 114, 171103

Aguilar M., et al., 2016, Physical Review Letters, 117, 231102

Aguilar M., et al., 2017, Phys. Rev. Lett., 119, 251101

Ahn H. S., et al., 2008, Astroparticle Physics, 30, 133

Arfken G. B., Weber H. J., 2005, Mathematical methods for physicists 6th ed.. Elsevier, Boson \& Amsterdam

Berezinskii V. S., Bulanov S. V., Dogiel V. A., Ptuskin V. S., 1990, Astrophysics of cosmic rays. Amsterdam, Netherlands: North-Holland Blandford R., Eichler D., 1987, Phys. Rep., 154, 1

Case G., Bhattacharya D., 1996, A\&AS, 120, 437

Cowsik R., Wilson L. W., 1973, International Cosmic Ray Conference, 1, 500

Drury L. O. C., Strong A. W., 2017, A\&A, 597

Engelmann J. J., Ferrando P., Soutoul A., Goret P., Juliusson E., 1990, A\&A, 233, 96

Erlykin A. D., Wolfendale A. W., 2006, Astroparticle Physics, 25, 183

Evoli C., Gaggero D., Grasso D., Maccione L., 2008, jcap, 10, 018

Garcia-Munoz M., Simpson J. A., Guzik T. G., Wefel J. P., Margolis S. H., 1987, ApJS, 64, 269

Green D. A., 2015, MNRAS, 454, 1517

Green D. A., 2017, VizieR Online Data Catalog, 7278

Isichenko M. B., 1991, Plasma Physics and Controlled Fusion, 33, 795

Jansson R., Farrar G. R., 2012a, ApJ, 757, 14

Jansson R., Farrar G. R., 2012b, ApJL, 761, L11

Kalberla P. M. W., Kerp J., 2009, ARA\&A, 47, 27

Kissmann R., 2014, Astroparticle Physics, 55, 37

Lezniak J. A., 1976, Nuclear Instruments and Methods, 136, 299

Longair M. S., 2011, High Energy Astrophysics. Cambridge University Press

Merten L., Becker Tjus J., Fichtner H., Eichmann B., Sigl G., 2017, J. Cosmology Astropart. Phys., 6, 046

Ramaty R., Kozlovsky B., Lingenfelter R. E., Reeves H., 1997, The Astrophysical Journal, 488, 730

Sasaki M., Breitschwerdt D., Supper R., 2004, Astrophysics and Space Science, 289, 283

Simon M., Heinrich W., Mathis K. D., 1986, ApJ, 300, 32

Strong A. W., Moskalenko I. V., 1998, Astrophys. J., 509, 212

Strong A. W., Moskalenko I. V., 2001, International Cosmic Ray Conference, 5, 1942

Strong A. W., Moskalenko I. V., Ptuskin V. S., 2007, Annual Review of Nuclear and Particle Science, 57, 285 
Strong A. W., Moskalenko I. V., Porter T. A., Jóhannesson G., Orlando E., Digel S. W., 2010, GALPROP: Code for Cosmic-ray Transport and Diffuse Emission Production, Astrophysics Source Code Library (ascl:1010.028)

Thoudam S., 2006, MNRAS, 370, 263

Thoudam S., 2007, MNRAS, 378, 48

Thoudam S., Rachen J. P., van Vliet A., Achterberg A., Buitink S., Falcke H., Hörandel J. R., 2016, A\&A, 595, A33

Venkatesan A., Coleman Miller M., Olinto A. V., 1997, ApJ, 484, 323

Webb G. M., Axford W. I., Forman M. A., 1985, ApJ, 298, 684

Webber W. R., Villa T. L., 2016

Webber W. R., Soutoul A., Kish J. C., Rockstroh J. M., 2003, ApJS, 144, 153

\section{APPENDIX A: LOCAL AND ISOTROPIC COSMIC RAY DIFFUSION IN THE GALACTIC DISK}

Consider cosmic rays (CRs) injected impulsively at $t=0$, somewhere in the mid-plane of the Galactic disk. Employing a set of local cylindrical coordinates $(\varpi, \theta, z)$ centered on the source location, with $z$ the coordinate perpendicular to the disk mid-plane $z=0$, isotropic diffusion of the CRs with a constant diffusion coefficient $D_{\mathrm{cr}}$ is described for $t>0$ by the following equation for the CR density $n_{\mathrm{cr}}(\varpi, z, t)$ :

$$
\frac{\partial n_{\mathrm{cr}}(\varpi, z, t)}{\partial t}=\frac{D_{\mathrm{cr}}}{\varpi} \frac{\partial}{\partial \varpi}\left(\varpi \frac{\partial n_{\mathrm{cr}}(\varpi, z, t)}{\partial \varpi}\right)+D_{\mathrm{cr}} \frac{\partial^{2} n_{\mathrm{cr}}(\varpi, z, t)}{\partial z^{2}}
$$

Here we assumed axi-symmetry around the source location. This is a reasonable approximation as long as the source is not close to the edge of the Galaxy. We assume that CRs escape instantaneously once the reach the upper/lower edge of the CR halo, located at $z= \pm H_{\mathrm{cr}}$. This leads to the condition:

$$
n_{\mathrm{cr}}\left(\varpi, z= \pm H_{\mathrm{cr}}, t\right)=0
$$

Solution of this equation subject to condition (A2) proceeds by standard means: a Laplace Transformation with respect to time, followed by separation of the remaining independent variables $\varpi$ and $z$. Defining the Laplace Transform of $n_{\mathrm{cr}}(\varpi, z, t)$ as $\tilde{n}_{\mathrm{cr}}(\varpi, z, s)$ so that:

$$
\tilde{n}_{\mathrm{cr}}(\varpi, z, s)=\int_{0}^{\infty} \mathrm{d} t e^{-s t} n_{\mathrm{cr}}(\varpi, z, t),
$$

standard theory, see for instance (Arfken \& Weber 2005), Ch. 15.8 yields:

$$
\frac{1}{\varpi} \frac{\partial}{\partial \varpi}\left(\varpi \frac{\partial \tilde{n}_{\mathrm{cr}}(\varpi, z, s)}{\partial \varpi}\right)+\frac{\partial^{2} \tilde{n}_{\mathrm{cr}}(\varpi, z, s)}{\partial z^{2}}-\left(\frac{s}{D_{\mathrm{cr}}}\right) \tilde{n}_{\mathrm{cr}}(\varpi, z, s)=0 .
$$

We seek a solution of the form

$$
\tilde{n}_{\mathrm{cr}}(\varpi, z, s)=R(\varpi, s) Z(z) .
$$

In order to satisfy (A3) we must demand $Z\left(z= \pm H_{\mathrm{cr}}\right)=0$ and look for solutions where, introducing the separation constant $-k^{2}$

$$
\frac{1}{Z} \frac{\mathrm{d}^{2} Z}{\mathrm{~d} z^{2}}=-k^{2}
$$

and

$$
\frac{1}{\varpi} \frac{\mathrm{d}}{\mathrm{d} \varpi}\left(\varpi \frac{\mathrm{d} R}{\mathrm{~d} \varpi}\right)-\left(k^{2}+\frac{s}{D_{\mathrm{cr}}}\right) R=0
$$

The solutions of (A6) that satisfy the condition $Z\left(z= \pm H_{\mathrm{cr}}\right)=0$ are: 


$$
Z(z)=\cos \left(k_{m} z\right), \quad k_{m} \equiv\left(m+\frac{1}{2}\right) \frac{\pi}{H_{\mathrm{cr}}} \text { with } m=0,1,2, \cdots
$$

The corresponding solutions of (A7) that satisfy $n_{\mathrm{cr}}(\varpi, z, t)=0$ for $\varpi \rightarrow \infty$ are:

$$
R(\varpi, s)=K_{0}\left(q_{m}(s) \varpi\right), q_{m}(s) \equiv \sqrt{k_{m}^{2}+\frac{s}{D_{\mathrm{cr}}}} .
$$

The general solution of (A4) is therefore:

$$
\tilde{n}_{\mathrm{cr}}(\varpi, z, s)=\sum_{m=0}^{\infty} a_{m} K_{0}\left(q_{m}(s) \varpi\right) \cos \left(k_{m} z\right)
$$

where the constant coefficients $a_{m}$ must be determined from the initial conditions. Their value need not concern us in what follows.

The CR density $n_{\mathrm{cr}}(\varpi, z, t)$ follows from the inverse Laplace Transform. For that we may use (c.f. Abramowitz \& Stegun (1972), p. 1028):

$$
K_{0}\left(a \sqrt{s+s_{0}}\right) \Longrightarrow \frac{e^{-s_{0} t}}{2 t} \exp \left(-\frac{a^{2}}{4 t}\right)
$$

In this particular case $a=\varpi / \sqrt{D_{\text {cr }}}$ and $s_{0}=k_{m}^{2} D$ and one finds:

$$
n_{\mathrm{cr}}(\varpi, z, t)=\frac{1}{2 t} \sum_{m=0}^{\infty} a_{m} \cos \left(k_{m} z\right) e^{-k_{m}^{2} D t} \exp \left(-\frac{\varpi^{2}}{4 D t}\right) .
$$

At late times, well after injection when the initial build-up of the density $n_{\mathrm{cr}}(\varpi, z, t)$ has passed, the $m=0$ term dominates (unless $a_{0}=0$, which is generally not the case) because of the exponential decay factor $\exp \left(-k_{m}^{2} D_{\text {cr }} t\right)$. Near the mid-plane of the disk $(z=0)$ and at a distance $\varpi$ from the source the CR density due to this single, impulsive source then roughly scales as:

$$
\begin{aligned}
n_{\mathrm{cr}}(\varpi, 0, t) & \propto \frac{1}{t} \exp \left[-\left(\frac{\pi^{2} D_{\mathrm{cr}} t}{4 H_{\mathrm{cr}}^{2}}+\frac{\varpi^{2}}{4 D_{\mathrm{cr}} t}\right)\right] \\
& =\frac{\pi D_{\mathrm{cr}}}{\varpi H_{\mathrm{cr}}} \frac{1}{\tau} \exp \left[-\frac{\pi \varpi}{4 H_{\mathrm{cr}}}\left(\tau+\frac{1}{\tau}\right)\right] .
\end{aligned}
$$

Here the dimensionless time variable $\tau$ is defined as:

$$
\tau=\frac{\pi D_{\mathrm{cr}} t}{\varpi H_{\mathrm{cr}}}
$$

so that the argument of the exponential factor in expression (A13) has a minimum at $\tau=1$. If we write that factor as $\exp (-\psi)$ we have $\psi_{\mathrm{min}}=\pi \varpi / 2 H_{\mathrm{cr}}$. The maximum value of $n_{\mathrm{cr}}(\varpi, 0, t)$ is reached $\tau=\tau_{\mathrm{m}}\left(\right.$ time $\left.t=t_{\mathrm{m}}\right)$ with

$$
\tau_{\mathrm{m}}(\varpi)=\frac{2 H_{\mathrm{cr}}}{\pi \varpi}\left(\sqrt{1+\frac{\pi^{2} \varpi^{2}}{4 H_{\mathrm{cr}}^{2}}}-1\right), \quad t_{\mathrm{m}}(\varpi)=\frac{4 t_{\mathrm{res}}}{\pi^{2}}\left(\sqrt{1+\frac{\pi^{2} \varpi^{2}}{4 H_{\mathrm{cr}}^{2}}}-1\right) .
$$

Here we have used $t_{\mathrm{res}}=H_{\mathrm{cr}}^{2} / 2 D_{\mathrm{cr}}$ for the typical CR residence time in the Galaxy. Note that $\tau_{\mathrm{m}} \simeq 1$ when $\varpi \gg H_{\mathrm{cr}}$ so that the exponential factor indeed dominates temporal behavior in that limit. Concluding: at given $\varpi$ and at late times (i.e. for $\tau \gg 1$ ) the local CR density in the mid-plane, at time $t$ after injection by an impulsive CR source at $\varpi=0$, decays roughly as 


$$
n_{\mathrm{cr}}\left(\varpi, 0, t=\frac{\varpi H_{\mathrm{cr}}}{\pi D_{\mathrm{cr}}} \tau\right) \propto \frac{\pi D_{\mathrm{cr}}}{\varpi H_{\mathrm{cr}} \tau} \exp \left(-\frac{\pi \varpi}{4 H_{\mathrm{cr}}} \tau\right) .
$$

This confirms the intuitive notion that CR escape limits the spread of diffusing CRs in the disk plane to a typical distance $\varpi \simeq \sqrt{2 D_{\text {cr }} t_{\text {res }}}=H_{\text {cr }}$ from the source when diffusion is isotropic. 